\title{
Determinación del nivel medio del mar en el mar de Bransfield. Aplicación a la determinación del geoide en islas Decepción y Livingston, Islas Shetland del Sur (Antártida)
}

\section{Determination of the Mean Sea Level in the Bransfield Sea. Application to determination of the geoid at Deception and Livingston islands (Antarctica)}

\author{
Bismarck Jigena Antelo? \\ Manuel Berrocoso Domínguez ${ }^{2}$ \\ Juan Manuel Vidal Pérez ${ }^{3}$
}

Recibido 15 de agosto de 2020; aceptado 20 de octubre 2020

\section{RESUMEN}

Este trabajo presenta un estudio detallado para la determinación del nivel medio del mar (NMM), las constituyentes de marea y un geoide de precisión en las islas Decepción y Livingston y el entorno del mar de Bransfield. Para realizar estos trabajos es necesario definir el marco de referencia geodésico, para ello se han utilizaron las redes geodésicas REGID (Red Geodésica de Isla Decepción), RENID (Red de Nivelación de Isla Decepción) y REGRID (Red Gravimétrica de Isla Decepción), utilizando como vértices fundamentales algunos puntos pertene-cientes a la red RGAE (Red Geodésica Antártica Española). Los datos de marea fueron obtenidos mediante sensores de presión instalados en las estaciones mareográficas de LIVMAR y DECMAR, islas Livingston y Decepción respectivamente, ubicadas muy cerca de las bases antárticas y Gabriel de Castilla. Se obtuvieron las primeras series temporales con 798 días de observación en

Departamento de Ciencias y Técnicas de la Navegación y Construcciones Navales, Universidad de Cádiz, España, correo electrónico: bismarck.jigena@gm.uca.es. ORCID o000-0002-4858-3415

Facultad de Ciencias, Laboratorio de Astronomía, Geodesia y Cartografía, Universidad de Cádiz, España, correo electrónico: manuel.berrocoso@uca.es. ORCID 0000-0002-1878-9658 
ambas estaciones, cuyo análisis armónico nos proporcionó 70 constituyentes de marea, siendo 19 las más representativas con amplitudes superiores a $1 \mathrm{~cm}$. Se determinamos el nivel medio del mar, utilizando como puntos fundamentales el LNOO (isla Decepción) y BEJC (isla Livingston). Por último, se determinó el modelo de geoide de precisión, GeoiDEC14, calculado a partir de mediciones GPS, gravimétricas y de nivelación y cuyo resultado ha sido contrastado con los modelos globales más actuales. Estos son los primeros resultados de determinación del nivel medio del mar y la obtención de un modelo de geoide de precisión en esta zona de la Antártida y mejoran trabajos previos realizados en la región.

Palabras clave: ondulación del geoide, gravimetría, nivelación, constituyentes de marea, Base Antártica Española.

\section{ABStract}

This work presents a detailed study in order to determine the mean sea level (NMM), the tidal constituents and a precision geoid at Deception and Livingston Islands and the Bransfield Sea area. To carry out these works, it is necessary to define the geodetic reference frame, for this the geodetic networks like REGID (Geodetic network of Deception Island), RENID (Levelling network of Deception Island) and REGRID (Gravimetric network of Deception Island) have been used. In addition, some geodesic points belonging to the RGAE network (Spanish Antarctic Geodetic network) as fundamental vertices, were used. The tidal data were obtained using pressure sensors installed at the LIVMAR and DECMAR tidal stations, Livingston and Deception islands, respectively, located very close to the Antarctic Bases and "Juan Carlos I" and Gabriel de Castilla. The first time series were obtained with 798 days of observation in both stations, whose harmonic analysis provided us with 70 tidal constituents, 19 being the most representative with amplitudes greater than $1 \mathrm{~cm}$. With these tidal series we determine the mean sea level, using the LNOO (Deception Island) and BEJC (Livingston island) as a fundamental points. Finally, the precision geoid model, GeoiDEC14, was determined from GPS, gravimetric and leveling measurements. To contrast the results, a comparison of GeoiDEC14 against the most current global models, was made. These are the first results of determining the mean sea level and obtaining a precision geoid model in this Antarctic area. The results obtained improve previous work carried out in the region.

Key words: Geoid undulation, gravimetry, leveling, tide constituents, Spanish Antarctic Base. 


\section{Introducción}

Las Islas Shetland del Sur, al ser parte de la Antártida, es una zona geográfica bastante extrema y con un entorno muy complejo para realizar observaciones oceanográficas y geofísicas en el terreno. Las observaciones de marea en la Antártida son muy escasas y las existentes corresponden a registros durante el verano austral, pues el resto del año las aguas de las zonas costeras normalmente permanecen congeladas (Dragani et al., 2004), y no se conocen datos que lleguen al año de duración marea (King \& Padman, 2005; Vidal et al., 2012). Hasta la fecha, para muchos estudios oceanográficos y geofísicos, se han utilizado datos obtenidos indirectamente, como las cartas cotidales, cartas de iso-amplitud y datos de altimetría por satélites que son muy generales. Es muy importante obtener una serie temporal de datos de mareas en base a medidas directas y con una duración mayor a un año, esto nos permite estudiar con datos reales, la propagación y características de la onda en el entorno de la estación y extrapolar estos resultados a lugares próximos.

Las primeras observaciones de los niveles del mar en la isla Decepción se realizaron en 1970, por personal del Servicio de Hidrográfía Naval de Argentina (SHN, 1970). Posteriormente, en 1991 el SHN, volvió a tomar datos de marea en este mismo lugar, Dragani et al. (2004). Con estos datos se obtuvo el primer datum vertical referido al vértice geodésico BARG (Base Argentina), cuyo valor de altura ortométrica fue de $2547 \mathrm{msnm}$. Este datum vertical fue trasladado mediante nivelación geométrica de primer orden geodésico hasta el punto LN00, ubicado en la Base Española Gabriel de Castilla, que ha sido utilizado como referencia vertical para la red RENID (Red de Nivelación de Isla Decepción) a partir del año 2003 y cuya cota ortométrica fue de 5430 msnm. A partir de este datum vertical se dotó de cota de nivelación los puntos pertenecientes a las líneas de nivelación de la red RENID (Berrocoso et al., 2006a, 2006b, 2008).

En cuanto a otros estudios relacionados con los niveles del mar, diversos autores utilizaron estaciones hidrográficas, mareógrafos y correntómetros para estimar la circulación y el transporte en la cuenca oriental del Estrecho de Bransfield y fueron instalados en las islas Low, Rey Jorge y Livingston (Lopez et al., 1993; 1994, 1999; Smithson, 1992; Schöne, 1998; Speroni, 2000; Padman et al., 2002; Wilmott et al., 2007; Vidal et al., 2012). Los estudios realizados determinaron que las mareas en el Estrecho de Bransfield tienen una combinación de frecuencias diurnas y semi-diurnas, obteniendo las componentes principales de mareas 01, K1, M2 y S2 y la variación de los rangos máximos de marea entre 1.7 m y 2.1 m (Dragani et al., 2004). Con los trabajos de Vidal et al. (2012), se obtuvieron los primeros resultados del nivel medio del mar con observaciones directas de 81 días en las estaciones DECMAR y LIVMAR.

El primer geoide en la Antártida fue presentado en 1980 por Geoscience Australia, por encargo del SCAR (Comité Científico de Investigación Antártica) calculado a partir del modelo geopotencial GEM10C, con curvas de $\mathrm{N}$ cada 20 metros. La segunda edición de este modelo fue elaborada en 1990 con 
curvas de $\mathrm{N}$ a intervalos de 5 metros, utilizando el elipsoide GRS80 y los coeficientes geopotenciales del modelo OSU89A (SCAR, 1993). Desde 1988, grupos de investigación argentinos y españoles han realizado un seguimiento sistemático de las actividades volcánicas y tectónicas en las islas Decepción y Livingston utilizando técnicas de geofísica y geodesia. Desde ese año también se comenzaron a establecer las primeras estaciones de la red RGAE y REGID con la finalidad de estudiar la actividad geodinámica en el volcán Decepción y su entorno (Berrocoso et al., 2006a, 2006c, 2008). En la vigilancia realizada a partir de esa fecha, se han detectado dos crisis volcánicas importantes en Decepción, en diciembre y enero de 1991 y en enero y febrero de 1999. En 1992, para la elaboración del mapa topográfico de isla Decepción a escala 1:25 000, el Centro Geográfico del Ejército Español (CGE) estableció como valor inicial para la ondulación del geoide (N) un valor convencional de 13 metros (CGE 1992). Este mapa junto a la ortoimagen de Isla Decepción a escala 1:20 000, son referentes cartográficos para la comunidad científica (Berrocoso et al., 2012).

Se establecieron dos bases gravimétricas que han permitido el enlace de las observaciones con el continente sudamericano y también de las observaciones marinas con la red gravimétrica terrestre en Ushuaia (Argentina), Punta Arenas (Chile), BAE "Juan Carlos I" (isla Livingston), Base Gravimétrica Argentina y Base Gravimétrica Española en isla Decepción (Carbó et al., 2001; Berrocoso et. al., 2007, 2008). Los trabajos de Carbó et al. (2001), dieron como resultado los mapas de anomalías de Bouguer, tanto en Puerto Foster como en la parte exterior de isla Decepción.

El primer geoide experimental de isla Decepción fue calculado según los trabajos de (Berrocoso et al., 2007, 2008). El mapa de ondulaciones del geoide de este trabajo se encuentra disponible en el Sistema Web SIMAC (Sistema de Información Multidisciplinar de Apoyo Científico para Isla Decepción) y también es explicado en Torrecillas et al. (2006). Los valores de $\mathrm{N}$ de este primer geoide experimental fueron calculados con la cota ortométrica de $5.43 \mathrm{msnm}$ definida para LN00, obteniendo un valor medio 19.59 m para $\mathrm{N}$ en isla Decepción. Este valor tiene una diferencia de más de 6 metros respecto al valor definido por el CGE (1992), sus detalles se pueden ver en Berrocoso et al. (2006a, 2007, 2008, 2012).

En este trabajo se presentan los resultados de nuevas observaciones de mareas en las estaciones de DECMAR y LIVMAR, con series de más de dos años de observación y que tienen como objetivo la obtención de nuevas constituyentes de marea, la determinación del nivel medio del mar y la determinación de un modelo de geoide de precisión para esta zona de la Antártida.

\section{2. Área de estudio}

El área de estudio pertenece a la cuenca del Bransfield situada entre la Península Antártica y el archipiélago de las Shetland del Sur. Las Islas Livingston y Decepción, forman parte de este archipiélago y se encuentran localizadas en 
el límite norte del Estrecho, siguiendo aproximadamente la prolongación del eje de expansión de la cuenca NE-SO. La cuenca del Bransfield, geodinámicamente se encuentra asociada a un área muy compleja, comprende parte del Cono Sur del Continente Americano, el Pasaje de Drake, las islas Shetland del Sur y la Península Antártica. Esta complejidad se debe a la confluencia de dos placas tectónicas principales: la placa Sudamericana y la placa Antártica; y tres micro placas que interactúan, la de Scotia, Drake (ex-Phoenix) y la de las Shetland del Sur, que según varios autores tienen un movimiento tectónico de 1,0 cm/año (González-Ferrán, 1991; Smellie et al., 1995; Fernández-Ros, 2006; Solari et al., 2008; Taylor et al., 2008).

El estrecho de Bransfield es una cuenca marginal activa en donde se ha identificado un eje de extensión con dirección NE-SW entre los paralelos $60^{\circ}$ y $63^{\circ}$ Sur, está protegido de mar abierto por las islas Smith, Snow y Livingston por el norte y al oeste, y por las islas Greenwich, Robert, Nelson y Rey Jorge, al norte y al este (Lenn et al., 2003). Está ocupada por seis edificios volcánicos alineados aproximadamente a lo largo de la dirección principal de la cuenca (Gracia et al., 1997). En Berrocoso et al. (2008) se dan más detalles sobre la tectónico regional.

Isla Decepción es un volcán activo, geológicamente muy joven, menos de 0,75 Ma (Valencio et al., 1979) y con erupciones históricas datadas en 1839, 1842, 1912, 1917, 1967, 1969 y 1970 (Smellie et al., 2002). La forma actual de la isla se debe al colapso de un enorme edificio volcánico, dejando una isla en forma de herradura con una caldera inundada, que ha formado una entrada de mar de 9-10 km de diámetro llamada Port Foster. Se comunica al estrecho de Bransfield a través de un paso angosto y poco profundo, de unos 500 metros denominado Fuelles de Neptuno (Smith et al., 2003). Una explicación detallada de las diferentes hipótesis de la formación de la isla y su bahía la podemos ver en Martí et al. (1996), Smellie (2001, 2002), Smellie et al. (2002), Fernández-Ros (2006) y Maestro et al. (2007).

En la isla se encuentran las bases antárticas "Gabriel de Castilla" (España) y "Decepción" (Argentina). Además, se encuentran las ruinas de la una exBase Chilena, cuyo funcionamiento terminó violenta y definitivamente el 4 de diciembre de 1967 con la última erupción volcánica, las ruinas de una factoría ballenera que estuvo operativa hasta 1931 y la base británica “Station B-Deception Island" abandonada el 23 de febrero de 1969. En la isla se encuentra instalada la estación mareográfica DECMAR, ubicada en Punta Colatinas a unos $3 \mathrm{~km}$ al sur de la BAE Gabriel de Castilla y aproximadamente a la misma distancia de los Fuelles de Neptuno.

La isla Livingston es la segunda isla en tamaño, ubicada al sur de archipiélago de las Islas Shetland, tiene unos $70 \mathrm{~km}$ de largo y un ancho variable de 4-32 km. En esta isla sin actividad volcánica se encuentran las bases antárticas "Juan Carlos I" (España), San Clemente de Ohrid (Bulgaria) y la base Shirreff (Chile). También está el Campamento Científico Livingston (Argentina) en la península Byers y la Cape Shirreff Field Station (USA). La estación mareográfica LIVMAR 
se encuentra instalada en Caleta Johnson muy cerca de la base "Juan Carlos I". Unos $20 \mathrm{~km}$ al sur de Livingston está la isla Decepción, siendo la distancia de navegación entre ambas bases de unos 50 kilómetros. La Figura 1 muestra el área de estudio y la ubicación de las estaciones mareográficas.

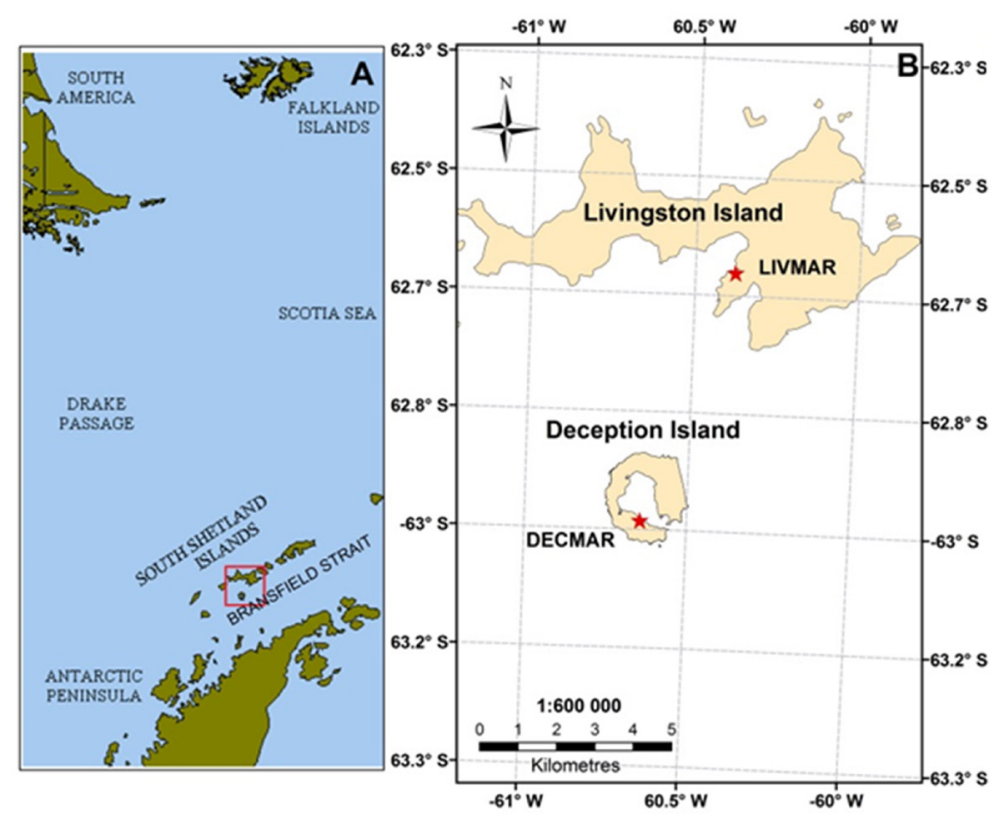

Figura 1. a. Área de estudio (polígono rojo), b. Islas Livingston y Decepción. Las estrellas rojas indican la ubicación de las estaciones mareográficas DECMAR and LIVMAR.

\section{Metodología, instrumentación y datos}

\subsection{Marco geodésico y gravimétrico}

El marco de referencia geodésico de nuestra área de estudio está compuesto por las diferentes redes geodésicas establecidas en la Antártida, entre las que se encuentran: Red Geodésica de la Antártida Española (RGAE), Red Geodésica de Isla Decepción (REGID), Red de Nivelación de Isla Decepción (RENID) y la Red Gravimétrica de Isla Decepción (REGRID), esta última constituye el marco gravimétrico de la isla.

Todas las estaciones de estas redes están dotadas de coordenadas geocéntricas tridimensionales $(X, Y, Z)$, transformadas a coordenadas geodésicas latitud $(\varphi)$, longitud $(\lambda)$ y altura elipsoidal (h) referidas al Marco Internacional de Referencia Terrestre ITRF2000.0 y ajustadas con un cierre de precisión 
milimétrico (Altamimi, Sillard y Boucher, 2002), como veremos más adelante. Para los trabajos cartográficos se han utilizado las coordenadas s planas rectangulares UTM (Universal Transversa de Mercator) para la Zona UTM 20 sur, Meridiano Central $63^{\circ}$ de longitud oeste.

\subsubsection{Red Geodésica de la Antártida Española (RGAE)}

La red RGAE fue la primera red diseñada en la Antártida por investigadores españoles, para definir un marco de referencia geodésico en la región de las Islas Shetland del Sur, el Mar de Bransfield y la Península Antártica (Figura 2).

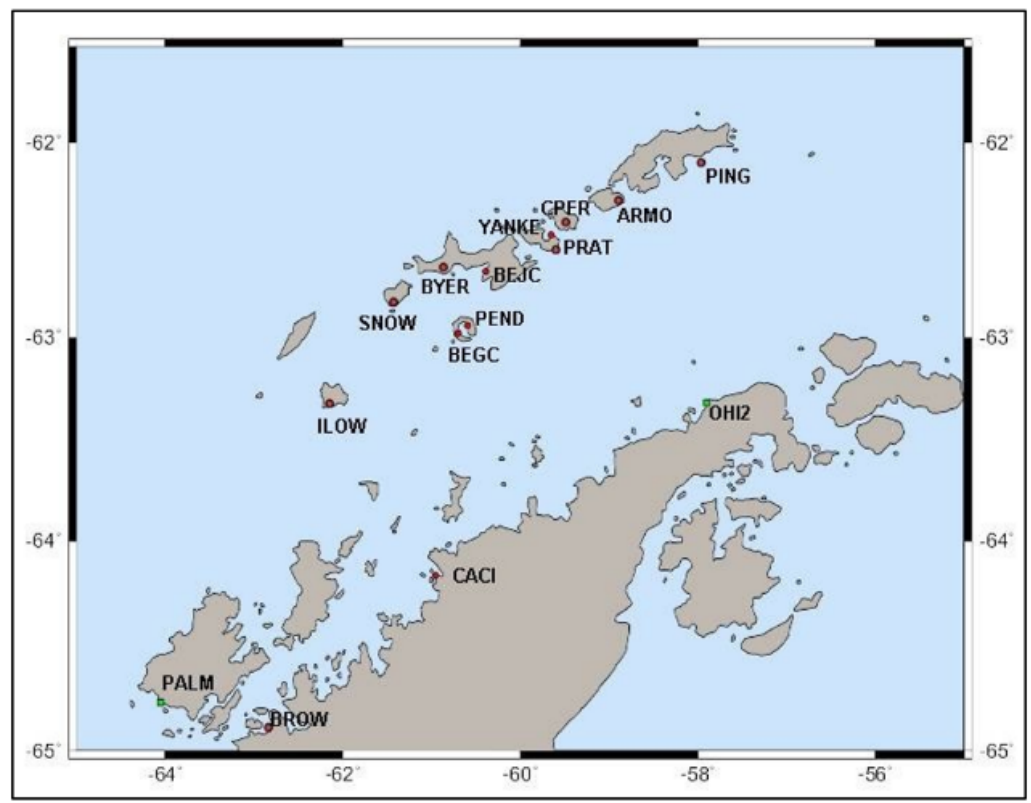

Figura 2. Red RGAE y la ubicación de sus vértices geodésicos.

Utilizando algunos vértices de esta red como puntos fundamentales, se determinaron las coordenadas de los vértices de la red REGID. Para este trabajo se utilizaron los siguientes: BEJC (BAE "Juan Carlos I", isla Livingston), BEGC (BAE Gabriel de Castilla, isla Decepción) PEND (Caleta Péndulo, isla Decepción). Mayores detalles de esta red geodésica (Berrocoso et al., 2006a, 2006b, 2006c, 2008).

\subsubsection{Red Ceodésica de Isla Decepción (RECID)}

La Red REGID es la segunda red geodésica establecida en la Antártida, específicamente en isla Decepción, con la finalidad de constituir el marco de 
referencia geodésico para cualquier actividad científica o técnica y establecer los puntos de control para determinar los modelos de deformación horizontal debidos a la actividad volcano-tectónica. La red está constituida por 13 vértices geodésicos distribuidos alrededor de Puerto Foster, que se pueden ver y en la Figura 3 y coordenadas ajustadas de la red se muestran en la Tabla 1. El benchmark geodésico LNOOO ha sido incluido en la red REGID, pues constituye el Punto Fundamental de Referencia Vertical de la red. Más detalles de la misma los podemos ver en Berrocoso et al. (2006a, 2006b, 2007, 2008).

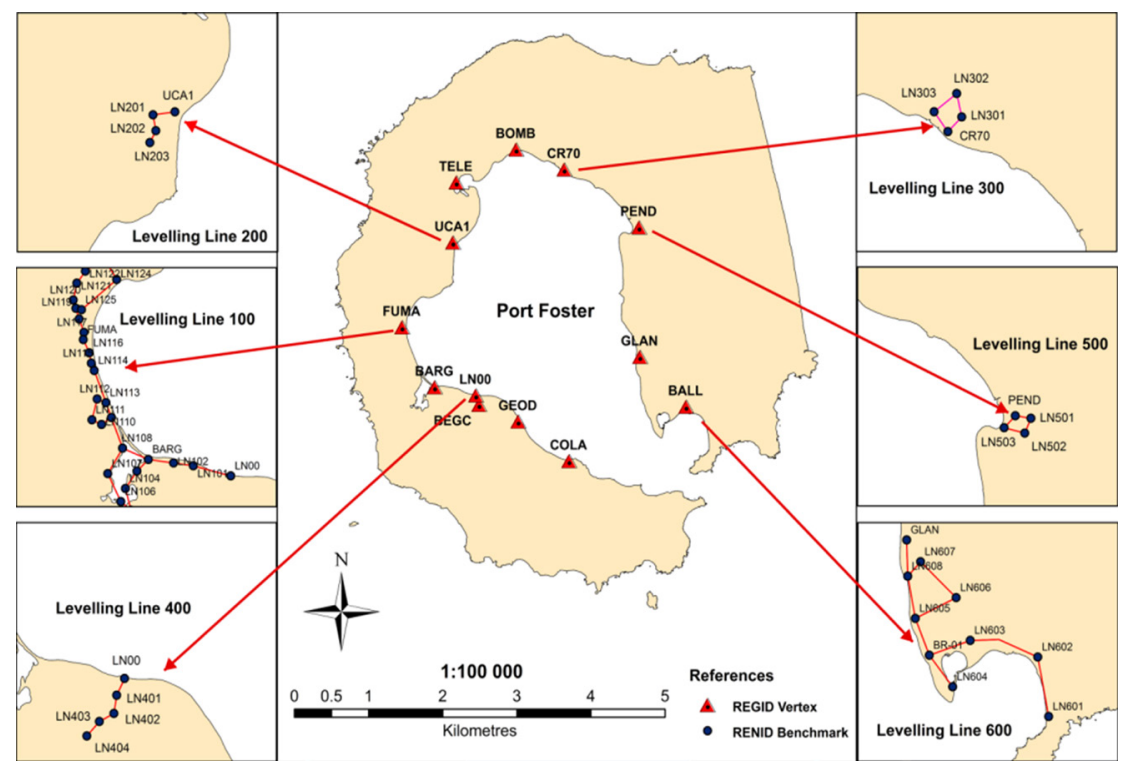

Figura 3. Red de Nivelación de Islas Decepción (RENID).

Tabla 1. Vértices red REGID. Coordenadas y precisiones (ITRF 2000.0)

\begin{tabular}{|c|c|c|c|c|c|c|c|c|c|c|c|}
\hline \multirow{2}{*}{$\frac{\text { No }}{1}$} & \multirow{2}{*}{$\frac{\text { VERTICE }}{\text { BEGC }}$} & \multicolumn{3}{|c|}{ Latitud S ( ${ }^{\circ}$ ' ") } & \multicolumn{3}{|c|}{ Longitud W( $\left(^{\circ}+"\right)$} & \multirow{2}{*}{\begin{tabular}{|c|}
$\mathbf{h}(\mathbf{m})$ \\
82.070
\end{tabular}} & \multirow{2}{*}{$\frac{\sigma \varphi(\mathbf{m})}{0.001}$} & \multirow{2}{*}{$\frac{\sigma \lambda(\mathbf{m})}{0.001}$} & \multirow{2}{*}{$\frac{\sigma h(\mathrm{~m})}{0.002}$} \\
\hline & & 62 & 58 & 43.6576 & 60 & 40 & 27.5304 & & & & \\
\hline 2 & BALL & 62 & 58 & 38.5553 & 60 & 33 & 52.5089 & 25.968 & 0.003 & 0.001 & 0.006 \\
\hline 3 & FUMA & 62 & 57 & 41.0170 & 60 & 42 & 59.3471 & 22.911 & 0.001 & $0.00 \mathrm{I}$ & 0.006 \\
\hline 4 & PEND & 62 & 56 & 09.8456 & 60 & 35 & 34.3437 & 28.841 & 0.001 & 0.001 & 0.003 \\
\hline 5 & COLA & 62 & 59 & 27.9180 & 60 & 37 & 31.8014 & 48.050 & 0.003 & 0.001 & 0.009 \\
\hline 6 & GLAN & 62 & 57 & 58.3621 & 60 & 35 & 23.8528 & 27.541 & 0.003 & 0.003 & 0.012 \\
\hline 7 & GEOD & 62 & 58 & 56.4127 & 60 & 39 & 11.7290 & 42.182 & 0.003 & 0.001 & 0.009 \\
\hline 8 & UCA1 & 62 & 56 & 28.4103 & 60 & 41 & 28.0891 & 28.667 & 0.003 & 0.001 & 0.009 \\
\hline 9 & CR70 & 62 & 55 & 23.6706 & 60 & 38 & 01.0013 & 23.600 & 0.003 & 0.003 & 0.015 \\
\hline 10 & TELE & 62 & 55 & 37.9905 & 60 & 41 & 25.5485 & 23.791 & 0.003 & 0.003 & 0.012 \\
\hline 11 & ВОМB & 62 & 55 & 08.4201 & 60 & 39 & 33.8481 & 23.785 & 0.003 & 0.003 & 0.015 \\
\hline 12 & BARG & 62 & 58 & 30.2679 & 60 & 41 & 53.3601 & 22.283 & 0.001 & 0.001 & 0.006 \\
\hline
\end{tabular}




\subsubsection{Red de Nivelación de Isla Decepción (RENID).}

La red de nivelación RENID consta de 60 marcas de nivelación (benchmarks), divididas en seis líneas de nivelación independientes.

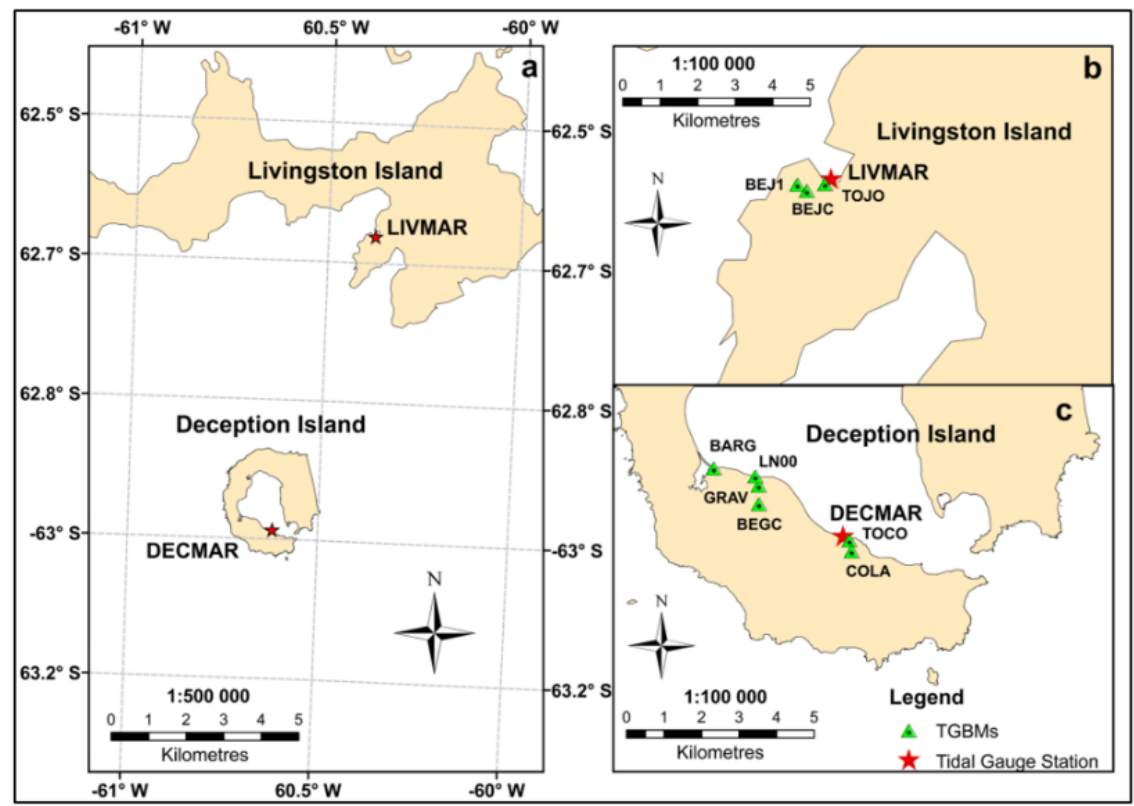

Figura 4. a. Ubicación de las estaciones mareográficas, b. Isla Livingston, ubicación de LIVMAR y sus correspondientes TGBM, c. Isla Decepción, ubicación de DECMAR y sus correspondientes TGBM.

El punto fundamental de referencia de altitud es el LNO0, situado junto a la Base Antártica Española "Gabriel de Castilla" y las líneas de nivelación que están vinculada al menos a uno de los vértices geodésicos de la red REGID, fueron establecidas en diferentes trabajos (Berrocoso et al. 2006a, 2006b, 2007, 2008; Vidal et al. 2012). La Figura 4 muestra las diferentes líneas de nivelación que componen la red RENID y los vértices geodésicos de la red REGID a los que están vinculados. En la Tabla 2 se muestran los datos de nivelación obtenidos en todos los puntos pertenecientes a la red RENID, así como los errores asociados a cada uno de ellos.

\subsubsection{Red Gravimétrica de isla Decepción, REGRID}


Para complementar las redes REGID y RENID en cuanto al seguimiento de la deformación vertical en isla Decepción, se estableció la red gravimétrica REGRID

Tabla 2. Puntos de la red RENID y con sus diferencias de nivel y sus errores asociados

\begin{tabular}{|c|c|c|c|c|c|c|c|}
\hline No & ESTACION & $\begin{array}{c}\Delta \mathrm{n} 2014 \\
(\mathrm{~m}) \\
\end{array}$ & $\begin{array}{c}\sigma \Delta \text { n } 2014 \\
(\mathrm{~m})\end{array}$ & No & ESTACION & $\begin{array}{c}\Delta \mathrm{n} 2014 \\
(\mathrm{~m})\end{array}$ & $\begin{array}{c}\sigma \Delta \mathrm{n} 2014 \\
(\mathrm{~m})\end{array}$ \\
\hline 1 & LN00 & 0.000 & 0.008 & 34 & LN119 & -1.94 & 0.020 \\
\hline 2 & BEGC & 57.025 & 0.003 & 35 & LN120 & -0.38 & 0.020 \\
\hline 3 & BALL & 0.723 & 0.026 & 36 & LN121 & 2.949 & 0.018 \\
\hline 4 & FUMA & -2.771 & 0.007 & 37 & LN122 & 5.776 & 0.018 \\
\hline 5 & PEND & 3.562 & 0.038 & 38 & LN123 & 12.02 & 0.013 \\
\hline 6 & COLA & 22.847 & 0.010 & 39 & LN124 & 0.051 & 0.008 \\
\hline 7 & GLAN & 2.213 & 0.033 & 40 & LN125 & -4.123 & 0.002 \\
\hline 8 & GEOD & 17.018 & 0.014 & 41 & LN201 & 17.845 & 0.009 \\
\hline 9 & UCA1 & 3.513 & 0.032 & 42 & LN202 & 6.567 & 0.011 \\
\hline 10 & CR70 & -1.531 & 0.042 & 43 & LN203 & 12.550 & 0.022 \\
\hline 11 & TELE & -1.394 & 0.068 & 44 & LN301 & 5.757 & 0.046 \\
\hline 12 & BOMB & -1.353 & 0.020 & 45 & LN302 & 19.817 & 0.050 \\
\hline 13 & GRAV & 23.456 & 0.000 & 46 & LN303 & -0.109 & 0.034 \\
\hline 14 & BARG & -2.883 & 0.008 & 47 & LN401 & 4.645 & 0.003 \\
\hline 15 & TOCO & -3.640 & 0.008 & 48 & LN402 & 12.409 & -0.013 \\
\hline 16 & LN101 & 2.136 & 0.003 & 49 & LN403 & 20.640 & -0.020 \\
\hline 17 & LN102 & 8.606 & 0.004 & 50 & LN404 & 32.454 & -0.012 \\
\hline 18 & LN103 & -0.756 & 0.008 & 51 & LN501 & -2.270 & 0.074 \\
\hline 19 & LN104 & -3.485 & 0.014 & 52 & LN502 & 19.396 & 0.056 \\
\hline 20 & LN105 & 4.960 & 0.009 & 53 & LN503 & 26.238 & 0.061 \\
\hline 21 & LN106 & 1.167 & 0.006 & 54 & BR-01 & 17.059 & 0.031 \\
\hline 22 & LN107 & 2.843 & 0.007 & 55 & LN601 & -4.347 & 0.054 \\
\hline 23 & LN108 & 0.266 & 0.000 & 56 & LN602 & 1.785 & 0.050 \\
\hline 24 & LN109 & 3.758 & 0.004 & 57 & LN603 & 8.617 & 0.043 \\
\hline 25 & LN110 & 20.362 & 0.003 & 58 & LN604 & -3.911 & 0.046 \\
\hline 26 & LN111 & 24.279 & 0.001 & 59 & LN605 & 3.668 & 0.048 \\
\hline 27 & LN112 & 10.539 & 0.009 & 60 & LN606 & 7.238 & 0.053 \\
\hline 28 & LN113 & -1.488 & 0.004 & 61 & LN607 & 12.875 & 0.074 \\
\hline 29 & LN114 & -4.002 & 0.008 & 62 & LN608 & 1.995 & 0.070 \\
\hline 30 & LN115 & -4.655 & 0.011 & & & & \\
\hline 31 & LN116 & -3.804 & 0.011 & 201 & BEJC & 0.000 & 0.000 \\
\hline 32 & LN117 & -3.74 & 0.012 & 202 & BEJ1 & 1.025 & 0.002 \\
\hline 33 & LN118 & -2.082 & 0.012 & 203 & TOJO & -9.482 & 0.000 \\
\hline
\end{tabular}

durante la Campaña Antártica 2002-03. Se realizó el enlace gravimétrico entre el continente sudamericano y las Islas Shetland del sur. El enlace se realizó desde la base gravimétrica APPA (Punta Arenas, Chile) hasta el vértice geodésico BEJC (BAE "Juan Carlos I" en la isla Livingston) y desde este se realizó el enlace con el vértice GRAV, definido como la Base Gravimétrica de Decepción (Berrocoso et al., 2008). Los datos obtenidos en estos enlaces se muestran en la Tabla 3. 
Tabla 3. Enlace gravimétrico entre el continente sudamericano y las islas Shetland del Sur (isla Livingston e isla Decepción)

\begin{tabular}{|c|c|c|c|}
\hline $\begin{array}{c}\text { Base Gravimetrica } \\
\text { (enlace) }\end{array}$ & $\begin{array}{c}\text { Valor Gravedad } \\
\text { (mGal) }\end{array}$ & $\begin{array}{c}\text { Desviación Estándar } \\
\text { (mGal) }\end{array}$ & $\begin{array}{c}\text { Número de } \\
\text { enlace }\end{array}$ \\
\hline APPA & 981320.8100 & 0.0150 & 1 \\
\hline BEJC & 982212.8190 & 0.1374 & 1 \\
\hline GRAV & 982202.5445 & 0.1866 & 1 \\
\hline
\end{tabular}

La red REGRID está compuesta por 12 estaciones geodésicas de la red REGID, 50 marcas de nivelación de la red RENID y 46 puntos gravimétricos auxiliares, en total 108 puntos. Todos estos puntos constituyen el marco de referencia gravimétrico en la isla y al cual están referidas todas las medidas de gravedad.

Esta red dispone además del pilar gravimétrico situado en el sótano de la antigua Base Argentina y de la nueva Base Gravimétrica de isla Decepción, el vértice GRAV, que constituye el Punto Fundamental de la red. En la Tabla 4 se muestran los puntos principales de la red RE-GRID con valores de gravedad y sus errores asociados. Más detalles en Jigena et al. (2016).

Tabla 4. Medidas red gravimétrica REGRID y sus errores asociados

\begin{tabular}{|c|c|c|c|c|c|c|c|c|c|c|c|}
\hline No & HSTACION & \begin{tabular}{|c|} 
GRAVEDAD \\
$(\mathrm{G} ; \mathrm{mGal})$
\end{tabular} & $\begin{array}{c}\sigma \mathrm{G} \\
(\mathrm{mG} \text { Gal) }\end{array}$ & No & HSTACION & \begin{tabular}{|c|} 
GRAVEDAD \\
$(\mathrm{G} ; \mathrm{mGal})$ \\
\end{tabular} & \begin{tabular}{|c|}
$\sigma G$ \\
$(\mathrm{mGal})$
\end{tabular} & No & ESTACION & \begin{tabular}{|c|} 
GRAVEDAD \\
(G; mGal)
\end{tabular} & $\begin{array}{c}\sigma \mathbf{G} \\
(\mathrm{mGal})\end{array}$ \\
\hline 1 & LN00 & 982206.408 & 0.051 & 22 & LN108 & 982206.612 & 0.102 & 43 & LN301 & 982200.833 & 0.056 \\
\hline 2 & BEGC & 982196.927 & 0.021 & 23 & LN109 & 982205.950 & 0.100 & 44 & LN302 & 982199.515 & 0.061 \\
\hline 3 & BALL & 982204.414 & 0.027 & 24 & LN110 & 982203.095 & 0.096 & 45 & LN303 & 982202.097 & 0.051 \\
\hline 4 & FUMA & 982204.912 & 0.026 & 25 & LN111 & 982202.109 & 0.089 & 46 & LN401 & 982206.461 & 0.053 \\
\hline 5 & PEND & 982202.237 & 0.022 & 26 & IN112 & 982204.271 & 0.077 & 47 & IN402 & 982205.851 & 0.064 \\
\hline 6 & COLA & 982205.580 & 0.035 & 27 & LN113 & 982206.870 & 0.059 & 4 & LN403 & 982204.553 & 0.065 \\
\hline 7 & GIAN & 982201.404 & 0.040 & 28 & INII4 & 982206.167 & 0.053 & 4 & IN404 & 982202.775 & 0.054 \\
\hline 8 & GEOD & 982203.666 & 0.028 & 29 & LN115 & 982206.008 & 0.061 & 5 & LN501 & 982203.602 & 0.071 \\
\hline 9 & UCA1 & 982201.949 & 0.029 & 30 & LN116 & 982205.614 & 0.054 & 5 & LN502 & 982200.069 & 0.075 \\
\hline 10 & CR70 & 982201.672 & 0.032 & 31 & LN117 & 982205.665 & 0.060 & 52 & LN503 & 982198.328 & 0.057 \\
\hline 11 & TELE & 982204.877 & 0.034 & 32 & LN118 & 982204.907 & 0.077 & 5. & BR-01 & 982200.614 & 0.053 \\
\hline 12 & BOMB & 982204.710 & 0.038 & 33 & LN119 & 982204.957 & 0.092 & 54 & LN601 & 982205.191 & 0.049 \\
\hline 13 & GRAV & 982202.545 & 0.006 & 34 & LN120 & 982204.608 & 0.094 & 55 & LN602 & 982204.490 & 0.051 \\
\hline 14 & BARG & 982206.942 & 0.018 & 35 & IN121 & 982204.254 & 0.092 & 56 & LN603 & 982203.097 & 0.052 \\
\hline 15 & LN101 & 982205.937 & 0.059 & 36 & LN122 & 982204.026 & 0.086 & 57 & LN604 & 982205.008 & 0.050 \\
\hline 16 & IN102 & 982204.751 & 0.052 & 37 & IN123 & 982203.253 & 0.075 & 58 & IN605 & 982202.019 & 0.066 \\
\hline 17 & LN103 & 982206.520 & 0.058 & 38 & LN124 & 982204.803 & 0.058 & 59 & LN606 & 982202.965 & 0.068 \\
\hline 18 & IN104 & 982207.415 & 0.077 & 39 & IN125 & 982205.356 & 0.087 & 60 & LN607 & 982200.233 & 0.050 \\
\hline 19 & LN105 & 982206.059 & 0.089 & 40 & LN201 & 982200.860 & 0.078 & 61 & LN608 & 982201.389 & 0.061 \\
\hline 20 & LN106 & 982207.936 & 0.096 & 41 & LN202 & 982202.898 & 0.078 & 62 & Toco & 982205.580 & 0.006 \\
\hline 21 & LN107 & 982205.767 & 0.100 & 42 & LN203 & 982202.238 & 0.087 & & & & \\
\hline
\end{tabular}

\subsection{Marco oceanográfico}

El marco oceanográfico en el área de estudio está definido por las estaciones mareograficas, puntos de control de mareógrafos (TGBM, en inglés: Tide Gauge 
benchmark), puntos auxiliares de referencia de mareógrafos (TGAR, en inglés: Tide Gauge Auxiliary Reference) y los vértices de referencia. Se instalaron dos estaciones mareográficas en las islas Livingston y Decepción denominadas LIVMAR y DECMAR, que se muestran en la Tabla 5.

Tabla 5. Estaciones mareográficas y sus marcas de referencia geodésicas, TGBM y TGAR

\begin{tabular}{|c|c|c|c|}
\hline Estaciones de Marea & Vértices Geodésicos & TGBM & TGAR \\
\hline LIVMAR & BEJ 1 & TOJO & CIJO \\
\hline DECMAR & COLA & TOCO & CINF, CSUP \\
\hline
\end{tabular}

La Figura 4 muestra la ubicación de las estaciones mareográficas, con sus respectivos Puntos de Control de Mareógrafos (TGBM) y sus referencias auxiliares (TGAR).

\subsection{Equipos utilizados para toma de datos}

Los datos de marea se obtuvieron mediante dos sensores de presión y temperatura SAIV modelo TD304, CTD 204 SAIV SD y AQUAlogger PT520. Los sensores de presión fueron fondeados a unos 30 metros de la línea de playa y a profundidades aproximadas de 7.5 y 3.5 metros en LIVMAR y DECMAR respectivamente. Para el fondeo se utilizó una embarcación neumática Zodiac Pro 500.

Las estaciones geodésicas tienen coordenadas absolutas con precisión milimétrica en $X, Y, Z$, y en latitud $(\varphi)$, longitud $(\lambda)$ y altura elipsoidal (h) relativas al Sistema de Referencia ITRF2000.00 (Altamimi et al., 2002). Para el posicionamento GPS se utilizaron receptores geodésicos Leica GX1230 y TRIMBLE 5700, en posicionamento estático (fase) con postprocesamiento y antena estándar, como se especifica en Seeber (2003).

La nivelación geométrica se realizó utilizando un nivel óptico Leica modelo NA2 con una precisión de $\pm 0.7 \mathrm{~mm}$ para un kilómetro de doble recorrido. Para las nivelaciones trigonométricas y los enlaces geodésicos, utilizamos la Estación Total Leica modelo TPS 403. A estas medidas realizadas se le aplicaron las correcciones por esfericidad terrestre y efectos de refracción atmosférica.

Las observaciones gravimétricas se realizaron con un gravímetro relativo LaCoste \& Romberg modelo D-203. Se aplicaron correcciones de mareas, altura y deriva a todo el conjunto de datos gravimétricos. Los detalles de los sensores y equipos utilizados los podemos ver en Jigena et al. $(2015,2016)$.

\section{Resultados y discusión}

\subsection{Determinación de las constituyentes de marea}


El período de registro de marea en ambas estaciones fue del 3 de febrero de 2011 al 11 de abril de 2013 (Figura 5). Todas las medidas de las series se normalizaron

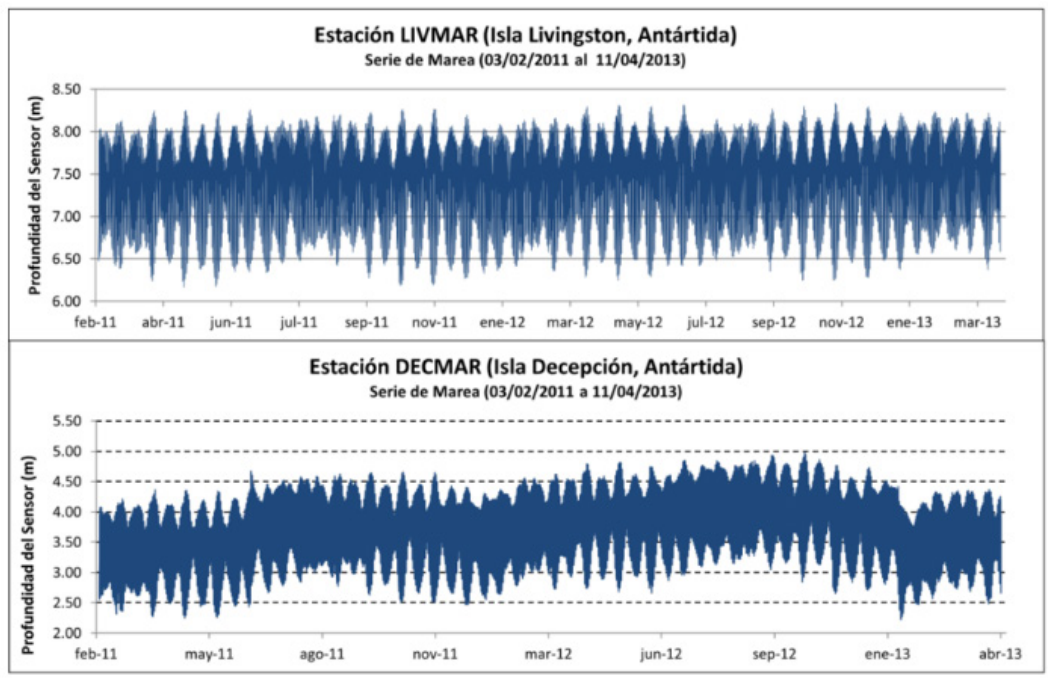

Figura 5. Series de mareas obtenidas en LIVMAR y DECMAR.

a 1 dato/hora. Las series temporales en cada estación fueron sometidas a un análisis armónico utilizando una aplicación desarrollada de acuerdo a Foreman (1977). Durante todo el período del registro de datos de marea, se obtuvieron datos meteorológicos de presión atmosférica y temperatura del aire, tomados por la Agencia Española de Meteorología (AEMET), en las estaciones de "Gabriel de Castilla" (isla Decepción) y "Juan Carlos I" (isla Livingston).

La presión atmosférica en la estación de isla Livingston se tomó como serie de referencia, al ser más estable y completa. La presión atmosférica en Livingston osciló entre un mínimo de 943,35 mb y un máximo de 1 025,59, con un promedio de 988,27 mb utilizado como referencia. Para convertir la presión hidrostática a una altura equivalente del nivel del mar, usamos:

$$
h=(P-P a) / g
$$

donde, P es la presión registrada por el sensor del mareógrafo, Pa es la presión atmosférica de referencia, utilizando el valor constante de 988,27 mb, g es la aceleración debida a la gravedad cuyo valor medio en Isla Decepción es 9,822956 $\mathrm{m} / \mathrm{s} 2$ y $\rho$ la densidad del agua en el área de estudio, cuyo valor medio es de 1025 kg/m3, siguiendo las especificaciones de la Organización de las Naciones Unidas para la Educación, la Ciencia y la Cultura (UNESCO, 1981, 1994) y Fofonot \& Millard (1983). Se realizó la corrección por efecto de barómetro invertido de acuerdo con Muñoz \& Abarca (2009). 
Las series de marea tienen un total de 19143 datos horarios, que supone un registro de más de dos años de observación (798 días) en ambas estaciones. En el análisis armónico obtuvimos una condición de matriz de 0.82 y una bondad de ajuste de 0.993 , teniendo como resultado 70 constituyentes de marea, siendo diecinueve (19) las más importantes, con amplitud mayor a un centímetro y un aporte de energía del 93\% del total de la onda. En todos los análisis armónicos realizados se obtuvo que la mayor cantidad de energía de la onda de marea (85\%) es aportada por ocho componentes, cuatro semidiurnas (M2, S2, K2, N2) y cuatro diurnas (K1, O1, P1, Q1), con un aporte similar para cada grupo de aproximadamente un 42,5\%. En lo referente a las amplitudes de las series, los resultados muestran que las amplitudes máximas y mínima son de $4986 \mathrm{~m}$. y $2224 \mathrm{~m}$ con un rango de $2762 \mathrm{~m}$ para DECMAR, y de $8334 \mathrm{~m}$ y $6169 \mathrm{~m}$ con un rango de $2165 \mathrm{~m}$ para LIVMAR. El factor de forma de marea o índice de Courtier (C) obtenido es 0,91 y 0.90 para LIVMAR y DECMAR respectivamente, determina que las mareas tienen un régimen mixto, con una componente predominantemente semidiurnas $(0.25<\mathrm{C}<1.50)$, definidas por Defant (1961), Dragani et al. (2004) y Vidal et al. (2012).

En general, los resultados en amplitud, desfase y régimen de marea, no difieren significativamente de los obtenidos por (Smithson, 1992; SCAR, 1993; López et al., 1993; García, 1994; Schöne et al., 1998; López et al., 1999; Speroni et al., 2000; Padman et al., 2002; D'onofrio et al., 2003; Dragani et al., 2004;

Tabla 6. Principales constituyentes de marea. Estaciones DECMAR y LIVMAR

\begin{tabular}{|c|c|c|c|c|c|c|c|}
\hline \multicolumn{4}{|c|}{ Estación LIVMAR } & \multicolumn{4}{|c|}{ Estación DECMAR } \\
\hline Constante & Frecuencia & $\begin{array}{l}\text { Amplitud } \\
\text { (m) }\end{array}$ & $\begin{array}{l}\text { Desfase } \\
\text { (grados) }\end{array}$ & Constante & Frecuencia & $\begin{array}{l}\text { Amplitud } \\
\text { (m) }\end{array}$ & $\begin{array}{c}\text { Desfase } \\
\text { (grados) }\end{array}$ \\
\hline $\mathrm{M} 2$ & 0.0805 & 0.39 & 290.1 & M2 & 0.0805 & 0.40 & 280.5 \\
\hline K1 & 0.0418 & 0.27 & 73.5 & K1 & 0.0418 & 0.28 & 68.9 \\
\hline 01 & 0.0387 & 0.26 & 54.9 & 01 & \begin{tabular}{ll|}
0.0387 \\
\end{tabular} & 0.27 & 52.5 \\
\hline S2 & 0.0833 & 0.20 & 351.2 & S2 & 0.0833 & 0.21 & 341.2 \\
\hline P1 & 0.0416 & 0.09 & 73.1 & P1 & 0.0416 & 0.09 & 68.5 \\
\hline Q1 & 0.0372 & 0.06 & 41.8 & $\mathrm{~K} 2$ & 0.0836 & 0.06 & 338.5 \\
\hline $\mathrm{K} 2$ & 0.0836 & 0.06 & 354.6 & Q1 & 0.0372 & 0.06 & 40.3 \\
\hline N2 & 0.0790 & 0.05 & 253.8 & $\mathrm{~N} 2$ & \begin{tabular}{l|l}
0.0790 \\
\end{tabular} & 0.05 & 245.1 \\
\hline $\mathrm{MF}$ & 0.0031 & 0.02 & 195.5 & SSA & 0.0002 & 0.04 & 324.9 \\
\hline NO1 & 0.0403 & 0.02 & 62.3 & MF & \begin{tabular}{l|l|}
0.0031 \\
\end{tabular} & 0.02 & 190.4 \\
\hline $\mathrm{MM}$ & 0.0015 & 0.02 & 128.5 & NO1 & 0.0403 & 0.02 & 51.7 \\
\hline $\mathrm{L} 2$ & 0.0820 & 0.01 & 308.9 & L2 & 0.0820 & 0.01 & 300.2 \\
\hline SSA & 0.0002 & 0.01 & 133.7 & $\mathrm{MM}$ & 0.0015 & 0.01 & 110.3 \\
\hline $\mathrm{J} 1$ & 0.0433 & 0.01 & 71.9 & $\mathrm{~T} 2$ & \begin{tabular}{l|l|}
0.0832 \\
\end{tabular} & 0.01 & 348.6 \\
\hline $\mathrm{T} 2$ & 0.0832 & 0.01 & 335.4 & SIG1 & 0.0359 & 0.01 & 37.0 \\
\hline RHO1 & 0.0374 & 0.01 & 49.4 & RHOI & 0.0374 & 0.01 & 39.6 \\
\hline SIG1 & 0.0359 & 0.01 & 37.9 & $\mathrm{~J} 1$ & 0.0433 & 0.01 & 68.5 \\
\hline $2 Q 1$ & 0.0357 & 0.01 & 29.2 & NU2 & 0.0792 & 0.01 & 243.6 \\
\hline NU2 & 0.0792 & 0.01 & 250.6 & $2 \mathrm{Q} 1$ & 0.0357 & 0.01 & 25.0 \\
\hline \multicolumn{2}{|c|}{ Longitud serie (días) } & \multicolumn{2}{|c|}{797.63} & \multicolumn{2}{|c|}{ Longitud serie (días) } & \multicolumn{2}{|c|}{797.63} \\
\hline \multicolumn{2}{|c|}{ Datos Observados } & \multicolumn{2}{|c|}{19143} & \multicolumn{2}{|c|}{ Datos Observados } & \multicolumn{2}{|c|}{19143} \\
\hline \multicolumn{2}{|c|}{ Condición de Matriz } & \multicolumn{2}{|c|}{0.82} & \multicolumn{2}{|c|}{ Condición de Matriz } & \multicolumn{2}{|c|}{0.82} \\
\hline \multicolumn{2}{|l|}{ Ajuste } & \multicolumn{2}{|c|}{0.993} & \multicolumn{2}{|l|}{ Ajuste } & \multicolumn{2}{|c|}{0.993} \\
\hline \multicolumn{2}{|l|}{ Courtier } & \multicolumn{2}{|c|}{0.91} & \multicolumn{2}{|l|}{ Courtier } & \multicolumn{2}{|c|}{0.90} \\
\hline
\end{tabular}


Vidal et al., 2012), sin embargo, se observan variaciones significativas en la constituyente $\mathrm{K} 1$ con respecto a los valores dados por (Dragani et al., 2004, Vidal et al., 2012). El resultado del ajuste entre los datos reales y los datos predichos es de un $99.3 \%$, que es un parámetro excelente y muy representativo para realizar predicciones en base a los datos obtenidos y utilizados en el análisis. Los resultados obtenidos en amplitud y desfase de las principales componentes de marea en ambas estaciones se muestran en la Tabla 6 y mayores detalles se pueden consultar en Jigena et al. (2015).

\subsection{Determinación del Nivel Medio del Mar y georreferenciación vertical}

Para el control vertical, los datos de marea fueron referidos altimétricamente a un TGBM, que a su vez está enlazado con otras marcas auxiliares de referencia de nivel TGAR y con el vértice geodésico más cercano, STGPS (en inglés: Station GPS). Toda la referenciación altimétrica se realizó mediante nivelación geométrica y el esquema de la misma lo podemos ver en la Figura 6. Las mediciones de control, entre el PTG (en inglés: Principal Tide Gauge) y el TSG (en inglés: Tide Staff Gauge) se realizaron en días escogidos, sin viento y sin olas (mar en calma) tomando las lecturas simultáneas al menos 1 hora, con una frecuencia de 10 minutos.

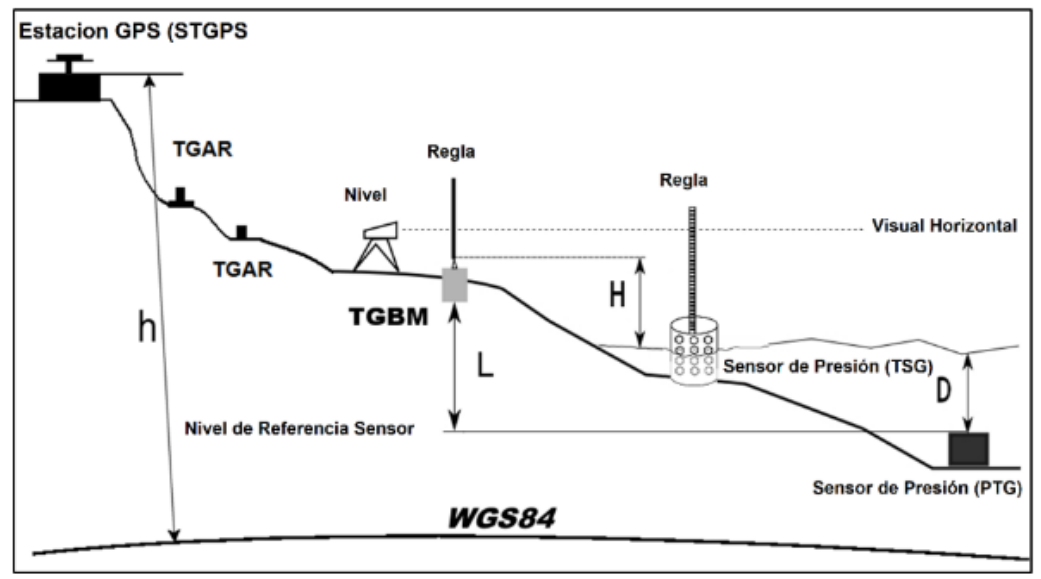

Figura 6. Vinculación altimétrica entre los TGBM (marca de referencia de mareógrafos), TGAR (marca auxiliar de referencia), PTG (sensor principal) y TSG (sensor móvil).

Fuente: adaptada de Jigena et al. (2015). 
Se realizó un ajuste lineal entre la lectura del sensor principal PTG, transformada a profundidad y corregida por presión atmosférica (D), contra los datos de altura $(\mathrm{H})$ tomados por el sensor de control TSG, instalado en la regla de marea y corregidos por la referencia al TGBM mediante nivelación geométrica. Este ajuste nos proporciona un valor medio del nivel de marea para cada instante de la lectura referido al TGBM y los valores medios de profundidad de fondeo de los sensores y cuyos resultados se aprecian en la Figura 7. Los detalles y la metodología se encuentran explicados en Vidal et al. (2012) y Jigena et al. $(2014,2015)$.

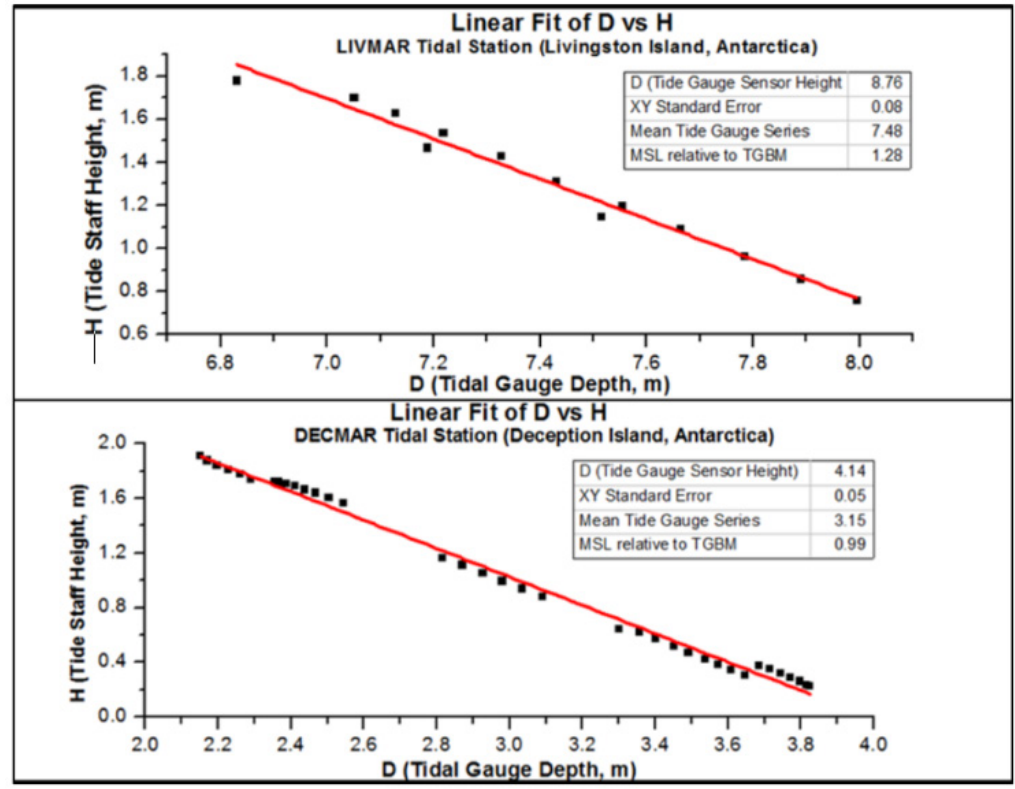

Figura 7. Ajuste lineal entre las medidas instantáneas del nivel medio del mar.

En la Tabla 7 vemos la relación entre las estaciones mareográficas con respecto a los vértices geodésicos de referencia, con los resultados obtenidos en los vértices de BEJC, COLA y LNO0, con los errores estimados asociados a los mismos. El valor obtenido del Nivel Medio del Mar (NMM) se corresponde con el valor de la altura ortométrica $(H)$ para el TGBM de referencia con su error correspondiente en la determinación del NMM para cada estación, para DECMAR (CINF) y para LIVMAR (CIJO).

Se obtuvo una altura ortométrica muy precisa en todos los puntos de control de mareógrafos (TGBM) y en los vértices geodésicos de referencia (BEJC, COLA y LNO0), corregida por efectos gravimétricos. La nivelación geométrica entre los TGBM, TGAR y los vértices geodésicos de referencia (STGPS) cumple con los estándares para nivelación geométrica de Primer Orden Geodésico. 
El benchmark LN00, fue definido como Punto Fundamental de Referencia Altimétrica de la red REGID y RENID, y su cota ortométrica queda determinada en 6200 msnm. En la Tabla 7 se muestran los resultados finales del cálculo de los valores del NMM, donde el valor calculado del error de $\mathrm{H}(\sigma \mathrm{H})$ incluye el error por medida y lectura del NMM. Además en la tabla h = altura alipsoidal; $\Delta \mathrm{n}=$ Diferencia de nivel (nivelación geométrica); $\mathrm{N}=$ Ondulación del geoide; $\mathrm{H}=$ Altura Ortométrica.

Tabla 7. Valores del Nivel Medio del Mar (NMM) y variables asociadas, en los vértices de referencia de las estaciones mareográficas DECMAR y LIVMAR

\begin{tabular}{|c|c|c|c|}
\hline \multirow{2}{*}{} & \multicolumn{2}{|c|}{ DECMAR (Isla Decepción) } & LVMAR (Isla Livingston) \\
\cline { 2 - 4 } & $\mathbf{C O L A}$ & $\mathbf{L N 0 0}$ & \multicolumn{1}{c|}{ BEJC } \\
\hline $\mathbf{h}$ (m) & $47.985 \pm 0.002$ & $25.069 \pm 0.002$ & $32.350 \pm 0.001$ \\
\hline $\mathbf{g}$ (mgal) & $982205.580 \pm 0.035$ & $982206.408 \pm 0.051$ & $982212.819 \pm 0.137$ \\
\hline Dn (m) & $29.047 \pm 0.003$ & $6.200 \pm 0.007$ & $12.418 \pm 0.003$ \\
\hline $\mathbf{N}$ (m) & $18.966 \pm 0.037$ & $18.932 \pm 0.056$ & $19.932 \pm 0.017$ \\
\hline $\mathbf{H}$ (m) & $\mathbf{2 9 . 0 5} \pm \mathbf{0 . 0 9}$ & $\mathbf{6 . 2 0} \pm \mathbf{0 . 1 1}$ & $\mathbf{1 2 . 4 2 \pm \mathbf { 0 . 1 0 }}$ \\
\hline
\end{tabular}

Las nuevas cotas ortométricas obtenidas tienen una diferencia $+0,06 \mathrm{~m}$ para BEJC y de + 0.20m para COLA, respecto a los valores obtenidos por (Vidal et al., 2012). En general, los resultados obtenidos en este trabajo han mejorado los trabajos anteriores realizados en la zona (SHN, 1970; Dragani et al., 2004; Vidal et al., 2012).

\subsection{Determinación del Geoide GEIODEC14}

Otro de los resultados de este trabajo, fue la determinación de un geoide experimental de precisión para la isla Decepción, denominado GeoiDEC14, además de tres puntos dotados con ondulación del geoide en isla Livingston, en el área aledaña a la BAE "Juan Carlos I" y a Caleta Johnsons. Para la determinación del geoide de precisión se utilizó la metodología GPS/Nivelación/ Gravedad, debido a que la misma computacionalmente es fácil de implementar, ya que simplemente se manejan vectores del tamaño de la cantidad de datos disponibles y el método se basa en la resolución de ecuaciones de segundo grado.

El método desarrollado está basado en la determinación de la ondulación del geoide (N) a partir de la diferencia entre la altura elipsoidal (h), obtenida directamente mediante observaciones GPS, y la altura ortométrica $(\mathrm{H})$ obtenida por nivelación geodésica, geométrica o trigonométrica y corregida por efectos gravimétricos mediante medidas de la gravedad (Heiskanen \& Moritz, 1985; Torge, 2001; Berrocoso et al., 1996), para ello utilizamos la fórmula que aproximadamente es igual a:

$$
h=H+N
$$


donde $h$ es la altura elipsoidal, $H$ la altura ortométrica y $N$ es la ondulación del geoide. Sabiendo que en un punto $\mathrm{P}$ de la superficie terrestre se conocen la cota ortométrica $(H)$ y la altura elipsoidal $(h)$, se puede determinar de forma muy aproximada la ondulación del geoide (N). La altura elipsoidal (h) la obtenemos directamente por observaciones GPS y partimos de un punto de altura ortométrica $(\mathrm{H})$ conocida, el problema para la aplicación del método se reduce a obtener la altura ortométrica en los demás puntos. Para calcular la altura ortométrica en un punto $\mathrm{PJ}_{\mathrm{J}}(\mathrm{HPj})$, obtenemos el siguiente desarrollo que nos permita calcular el de $\mathrm{H}$ partiendo de la diferencia de alturas ortométricas $\Delta \mathrm{HPi} ;$ j entre el punto de cálculo Pj y el punto de referencia Pi, y que nos dá como resultado la siguiente ecuación:

$$
0.0424^{*} 10^{-3} H_{P_{j}}^{2}+g_{P_{i}} H_{P_{j}}-\left(g_{P_{j}} \Delta n_{P_{i, j}}+g_{P_{i}} H_{P_{i}}+0.0424 * 10^{-3} H_{P_{i}}^{2}\right)=0
$$

Es una ecuación de segundo grado, donde la incógnita es la altura ortométrica $\mathrm{HP}_{\mathrm{j}}$ ) del punto $\mathrm{P}_{\mathrm{j}}$ que puede ser calculada a partir de la altura ortométrica $\mathrm{HP}_{\mathrm{i}}$ ) en el punto de referencia $\mathrm{P}_{\mathrm{i}}$ la diferencia de nivel $\Delta \mathrm{nP}_{\mathrm{i}, \mathrm{j}}$ entre los puntos $\mathrm{Pi}$ y $\mathrm{Pj}$ y los valores de la gravedad absoluta en estos puntos, $\mathrm{gP}_{\mathrm{i}}$ y $\mathrm{gP}_{\mathrm{j}}$.

Tabla 8. GeoiDEC14: Valores ondulación del geoide y sus errores estimados

\begin{tabular}{|c|c|c|c|c|c|c|c|c|c|c|c|}
\hline No. & ESTACION & $\begin{array}{c}N_{2014} \\
\text { (m) }\end{array}$ & $\begin{array}{c}\sigma \mathrm{N}_{2014} \\
\text { (m) }\end{array}$ & No. & ESTACION & $\begin{array}{c}N_{2014} \\
\text { (m) }\end{array}$ & $\begin{array}{c}\sigma \mathrm{N}_{2014} \\
\text { (m) }\end{array}$ & No. & ESTACION & $\begin{array}{c}N_{2014} \\
\text { (m) }\end{array}$ & $\begin{array}{c}\sigma N_{2014} \\
\text { (m) }\end{array}$ \\
\hline 1 & LN00 & 18.932 & 0.091 & 35 & LN122 & 18.592 & 0.119 & 69 & EG11 & 18.925 & 0.188 \\
\hline 2 & BEGC & 18.744 & 0.092 & 36 & LN123 & 18.092 & 0.110 & 70 & EG13 & 18.909 & 0.188 \\
\hline 3 & BALL & 19.156 & 0.119 & 37 & LN124 & 18.655 & 0.106 & 71 & EG14 & 18.928 & 0.188 \\
\hline 4 & FUMA & 19.485 & 0.100 & 38 & LN125 & 18.547 & 0.101 & 72 & G02 & 18.467 & 0.188 \\
\hline 5 & PEND & 19.082 & 0.129 & 39 & LN201 & 18.925 & 0.102 & 73 & G03 & 18.493 & 0.188 \\
\hline 6 & COLA & 18.916 & 0.106 & 40 & LN202 & 18.900 & 0.100 & 74 & G04 & 18.555 & 0.188 \\
\hline 7 & GLAN & 19.083 & 0.133 & 41 & LN203 & 18.878 & 0.112 & 75 & G07 & 18.829 & 0.188 \\
\hline 8 & GEOD & 18.905 & 0.111 & 42 & LN301 & 18.976 & 0.140 & 76 & G10 & 18.873 & 0.188 \\
\hline 9 & UCAI & 18.978 & 0.129 & 43 & LN302 & 18.921 & 0.144 & 77 & G14 & 19.309 & 0.188 \\
\hline 10 & CR70 & 18.963 & 0.145 & 44 & LN303 & 19.141 & 0.128 & 78 & G15 & 19.245 & 0.188 \\
\hline 11 & TELE & 18.986 & 0.168 & 45 & LN401 & 18.390 & 0.101 & 79 & G16 & 19.224 & 0.188 \\
\hline 12 & BOMB & 18.975 & 0.123 & 46 & LN402 & 18.610 & 0.114 & 80 & G17 & 18.831 & 0.188 \\
\hline 13 & GRAV & 18.897 & 0.098 & 47 & LN403 & 19.029 & 0.125 & 81 & G18 & 18.904 & 0.188 \\
\hline 14 & LN101 & 18.934 & 0.093 & 48 & LN404 & 18.721 & 0.116 & 82 & G20 & 18.837 & 0.188 \\
\hline 15 & LN102 & 18.888 & 0.095 & 49 & LN501 & 18.741 & 0.170 & 83 & G21 & 18.782 & 0.188 \\
\hline 16 & LN103 & 18.925 & 0.099 & 50 & LN502 & 18.525 & 0.157 & 84 & G22 & 18.845 & 0.188 \\
\hline 17 & LN104 & 18.958 & 0.108 & 51 & LN503 & 19.041 & 0.162 & 85 & G26 & 18.940 & 0.188 \\
\hline 18 & LN105 & 18.897 & 0.109 & 52 & BR-01 & 18.179 & 0.130 & 86 & G27 & 18.883 & 0.188 \\
\hline 19 & LN106 & 18.943 & 0.101 & 53 & LN601 & 18.682 & 0.155 & 87 & G28 & 19.014 & 0.188 \\
\hline 20 & LN107 & 18.920 & 0.100 & 54 & LN602 & 18.507 & 0.148 & 88 & G31 & 18.823 & 0.188 \\
\hline 21 & LN108 & 18.909 & 0.091 & 55 & LN603 & 18.712 & 0.144 & 89 & G32 & 18.861 & 0.188 \\
\hline 22 & LN109 & 18.910 & 0.095 & 56 & LN604 & 18.144 & 0.145 & 90 & G33 & 18.926 & 0.188 \\
\hline 23 & LN110 & 18.883 & 0.095 & 57 & LN605 & 18.275 & 0.147 & 91 & G34 & 18.905 & 0.188 \\
\hline 24 & LN111 & 18.890 & 0.098 & 58 & LN606 & 19.015 & 0.153 & 92 & G35 & 18.945 & 0.188 \\
\hline 25 & LN112 & 18.944 & 0.100 & 59 & LN607 & 18.349 & 0.177 & 93 & G42 & 18.986 & 0.188 \\
\hline 26 & LN113 & 18.939 & 0.099 & 60 & LN608 & 18.704 & 0.170 & 94 & $\mathbf{G 4 3}$ & 18.920 & 0.188 \\
\hline 27 & LN114 & 18.936 & 0.099 & 61 & EG01 & 19.962 & 0.188 & 95 & G44 & 18.880 & 0.188 \\
\hline 28 & LN115 & 18.611 & 0.108 & 62 & EG02 & 18.803 & 0.188 & 96 & G45 & 18.902 & 0.188 \\
\hline 29 & LN116 & 18.586 & 0.114 & 63 & EG03 & 19.085 & 0.188 & 97 & G48 & 18.986 & 0.188 \\
\hline 30 & LN117 & 18.958 & 0.105 & 64 & EG05 & 19.249 & 0.188 & 98 & G49 & 19.042 & 0.188 \\
\hline 31 & LN118 & 18.363 & 0.112 & 65 & EG06 & 18.874 & 0.188 & 99 & $\mathbf{G 5 3}$ & 18.910 & 0.188 \\
\hline 32 & LN119 & 18.053 & 0.119 & 66 & EG07 & 18.814 & 0.188 & 100 & G55 & 18.859 & 0.188 \\
\hline 33 & LN120 & 18.173 & 0.118 & 67 & EG09 & 18.941 & 0.188 & 101 & G57 & 18.872 & 0.188 \\
\hline 34 & LNI21 & 18.688 & 0.120 & 68 & EGIO & 18.888 & 0.188 & 102 & TOCO & 19.009 & 0.104 \\
\hline
\end{tabular}


Con estos datos se obtuvo el mapa de curvas de $\mathrm{N}$ aplicando el método interpolación para toda la isla (Figura 8a). Igualmente, se obtuvo el mapa de errores en metros de la ondulación del geoide (Figura 8b).
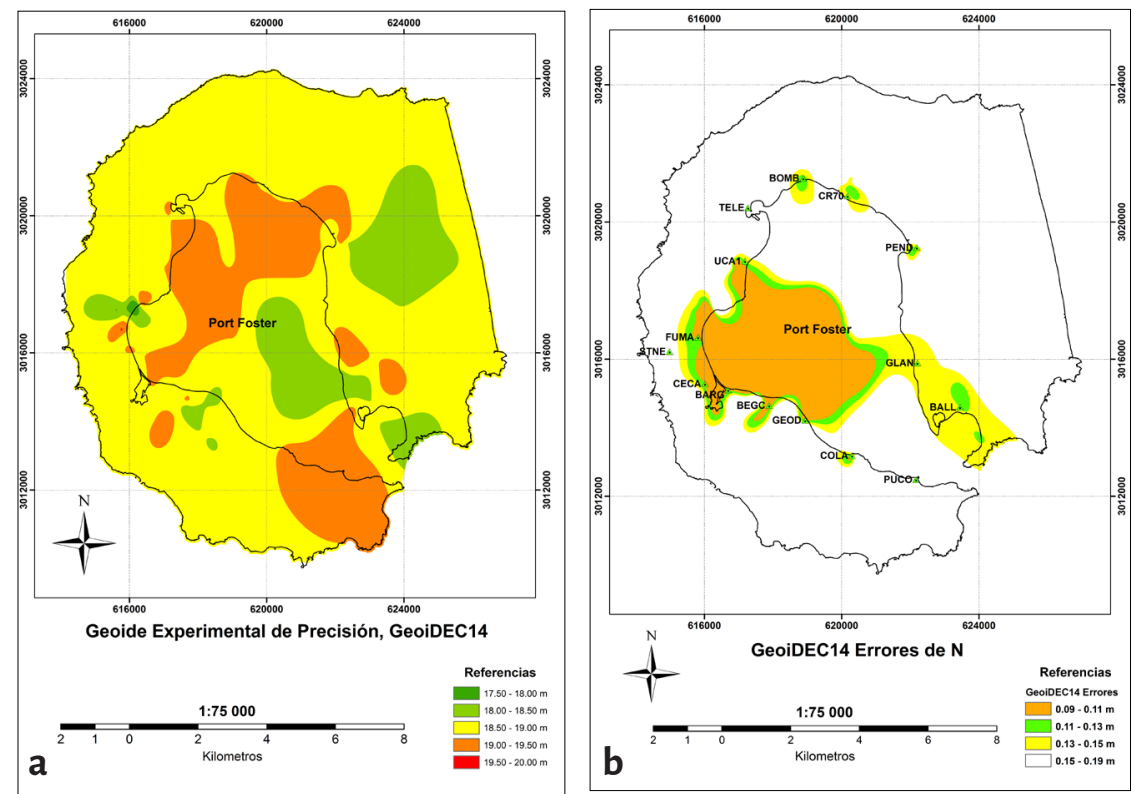

Figura 8. a. Valores de $\mathrm{N}$ obtenidos para GeoiDEC14, b. Errores estimados de N agrupados por zonas.

Observamos que los mayores errores se obtuvieron en las zonas Cráteres del 70, Caleta Péndulo y Punta Collins, mientras que en la zona de Bahía Balleneros se observó un cambio de tendencia. En general, los errores estimados en la determinación de GeoiDEC14 no exceden los 20 cm y pueden agruparse en tres tipos y están relacionados geográficamente a un área concreta de la isla como se muestra en la Tabla 9.

Tabla 9. Errores estimados del geoide GeoiDEC14, agrupados por zonas

\begin{tabular}{|l|c|}
\hline \multicolumn{1}{|c|}{ AREA } & ERROR (m) \\
\hline $\begin{array}{l}\text { Colatinas Point, Gabriel de Castilla Base, Argentine Base, } \\
\text { Fumarole Bay, Obsidianas, Pendulum Cove }\end{array}$ & $0.09-0.11$ \\
\hline Bombs Field, Craters 70, Black Glacier & $0.11-0.15$ \\
\hline Telephon Bay, Collins Point, Lobera Beach & $0.15-0.19$ \\
\hline
\end{tabular}


En la isla Livingston, área de la BAE "Juan Carlos I" y caleta Johnsons, se han establecido tres puntos provistos de ondulación del geoide $(\mathrm{N})$, altura ortométrica y coordenadas absolutas GNSS. En la Tabla 10 se muestran los resultados de estas observaciones en la isla Livingston y la ubicación de las estaciones se muestran en la Figura 4.

Tabla 10. Valores experimentales de $\mathrm{N}$ obtenidos en isla Livingston

\begin{tabular}{|c|c|c|c|c|c|}
\hline No & PUNTO & $\begin{array}{c}\mathbf{X} \\
(\mathbf{m})\end{array}$ & $\begin{array}{c}\mathbf{Y} \\
(\mathbf{m})\end{array}$ & $\begin{array}{c}\mathbf{N}_{\mathbf{2 0 1 4}} \\
(\mathbf{m})\end{array}$ & $\begin{array}{c}\sigma \mathbf{N} \\
(\mathbf{m})\end{array}$ \\
\hline 201 & BEJC & 633755.090 & 3049250.005 & 18.808 & 0.052 \\
\hline 202 & BEJ1 & 633803.084 & 3049258.486 & 18.905 & 0.054 \\
\hline 203 & TOJO & 633624.647 & 3049599.424 & 18.917 & 0.068 \\
\hline
\end{tabular}

La importancia de este trabajo radica en que es el primer modelo de un geoide local de precisión obtenido en una zona volcánica activa, como es el volcán Decepción y en esta zona de la Antártida. Este modelo permitirá realizar trabajos técnicos y científicos y producir resultados con un alto nivel de precisión. Como es un modelo preciso puede técnicamente tener la capacidad de ser utilizado para:

- la calibración de modelos geopotenciales globales,

- la determinación directa de alturas ortométricas combinando el modelo con observaciones GPS,

- en la elaboración de modelos de deformación y en monitoreo de la deformación producida por efectos volcánicos,

- en el diseño de modelos de flujo de lavas y en las previsiones para determinar mapas de peligrosidad y localización de las zonas de riesgo,

- en aplicaciones de oceanografía, control de los niveles del mar, topografía directa con GPS y en aplicaciones geofísicas.

Para obtener el modelo GeiDEC14, hemos utilizado la metodología GPS/ nivelación/gravedad realizando el cálculo sobre un total de 108 puntos disponibles en toda la isla, distribuidos homogéneamente en la parte interior de la isla alrededor de Puerto Foster. Estos puntos tienen un valor promedio de ondulación del geoide (N) de 18,83 metros con un rango de valores entre 18,05 y 19,49 metros.

Se obtuvieron tres puntos en isla Livingston, todos se encuentran ubicados en la zona entre la BAE "Juan Carlos I" y caleta Johnson. Estos tres puntos tienen un valor promedio de 18,87 metros, muy similar y coherentes con los obtenidos en isla Decepción. Debido a que estos puntos son muy cercanos su obtención 
no es relevante ni suficiente para la determinación de un geoide local en esta área; sin embargo, sirven para contrastar los datos obtenidos de GEO-DEC14 en Decepción.

Se ha realizado una comparación entre las ondulaciones del geoide obtenidas con modelos globales de geoide como el EGM96, EGM2008, AUIB-Grace2003s y ITSG-Grace2014s, además del geoide experimental de isla Decepción 2007 determinado por Berrocoso et al. (2007, 2008), contra las obtenidas con el GEOIDEC14, que nos han servido para comparar y analizar sus diferencias con respecto a nuestro geoide experimental. Los resultados se observan en la Figura 9c y Figura 9f, los valores de GeoiDEC14 se han introducido en cuadricula del EGM08 y GRACE2014s para mostrar su influencia en la generación de los modelos combinados. Podemos observar que GeoiDEC14 tiene valores más pequeños de N, llegando a los 18,05 metros en algunas zonas, valor no alcanzado por ninguno de los geoides globales estudiados.

La comparación muestra una diferencia máxima del valor de N, en los puntos observados de alrededor de 2,5 m con respecto a los antiguos modelos globales EGM96, GRACE03 y EGM2008 (Figuras 9a, 9b y 9d). Respecto a los modelos DEC2007 y GRACE2014 (Figura 9e) la diferencia es de 1,9 m.

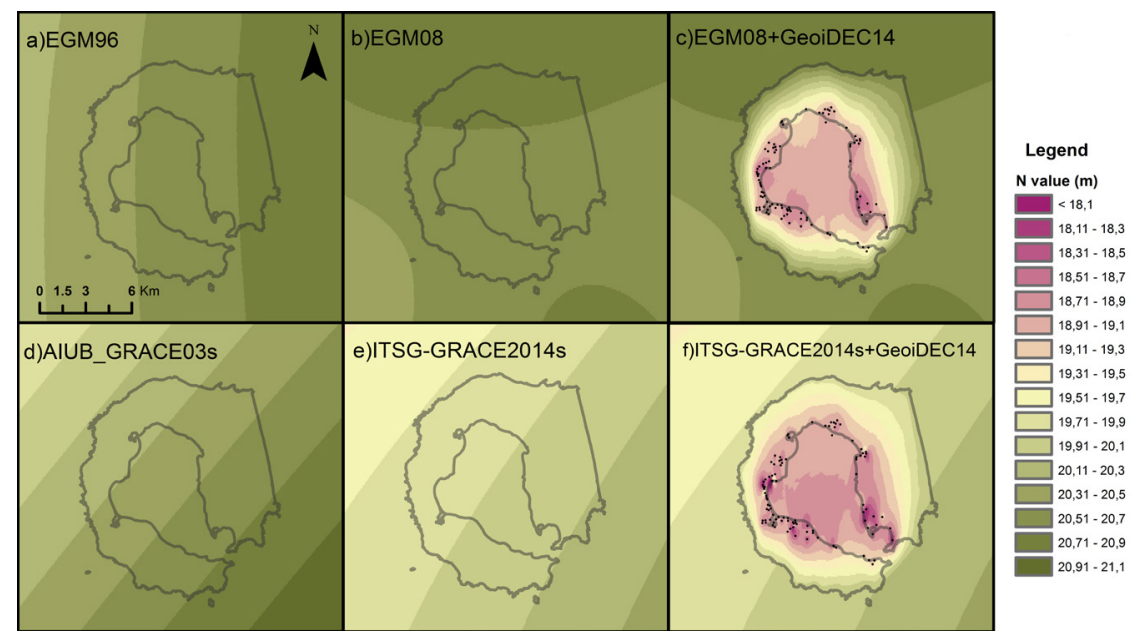

Figura 9. Valores de $\mathrm{N}$ obtenidos para a) EGM96, b) EGM08, d) AIUB_GRACE03s, e) ITSGGRACE2014s, c) and f) muestran los resultados de la inserción de GeoiDEC14 dentro de EGM08 y GRACE2014. 
En la Tabla 11 se presenta un análisis estadístico realizado, denominado Tamaño de Efecto (ES, en inglés Effect Size), que nos sirve para comparar diferentes modelos tomando uno de ellos como referencia, en este caso GEOIDEC14 (Ledesma et al., 2008). Los modelos donde ES es mínimo serían aquellos que se acerquen más a nuestro modelo patrón. Los resultados indican que los valores mínimos corresponden a los modelos GRACE2014 y DEC2007. Con este análisis nuevamente se marca una separación entre los modelos y se confirma que estos son los que tienen la mayor precisión y resolución entre los comparados. Este análisis validaría nuestra hipótesis de que el modelo de geoide local de precisión GeoiDEC14 es el modelo más preciso obtenido hasta la fecha en el área de la isla Decepción.

Tabla 11. Resumen estadístico de los valores de N (metros) entre los modelos geopotenciales globales y los modelos locales DEC2007 versus GeoiDEC14.

B. Diferencias de N entre modelos respecto a GeoiDEC14. ES_Max y ES_ Average es el resultado de aplicar el análisis Tamaño del Efecto (ES)

\begin{tabular}{|c|r|r|r|r|r|r|r|r|r|r|r|}
\hline & \multicolumn{4}{|c|}{ A. Resumen Estadistico } & \multicolumn{3}{c|}{ A. Diferencias de N respecto a (iEOIDEC14 } \\
\hline & GOIDEC14 & GEOIDEC07 & EGM96 & GRACE03 & LGM08 & GRACE14 & $\begin{array}{c}\text { Dif } \\
\text { LGM96 }\end{array}$ & $\begin{array}{c}\text { D Dif } \\
\text { GRACE03 }\end{array}$ & $\begin{array}{c}\text { Dif } \\
\text { EGM08 }\end{array}$ & \multicolumn{1}{c|}{$\begin{array}{c}\text { Dif } \\
\text { GRACE14 }\end{array}$} & $\begin{array}{c}\text { Dif } \\
\text { GEOIDEC07 }\end{array}$ \\
\hline MAX & 19.49 & 20.37 & 20.69 & 20.70 & 20.73 & 20.18 & -0.96 & -0.81 & -1.12 & -0.32 & 0.26 \\
\hline MIN & 18.05 & 18.77 & 20.44 & 20.23 & 20.57 & 19.78 & -2.51 & -2.52 & -2.57 & -1.90 & -1.85 \\
\hline PROMEDIO & 18.82 & 19.64 & 20.53 & 20.40 & 20.64 & 19.88 & -1.71 & -1.58 & -1.81 & -1.06 & -0.82 \\
\hline SD & -0.26 & \pm 0.28 & \pm 0.08 & \pm 0.13 & \pm 0.05 & \pm 0.08 & \pm 0.28 & -0.30 & \pm 0.26 & \pm 0.28 & \pm 0.23 \\
\hline ES_Max & 0.00 & 4.56 & 4.58 & 4.69 & 2.25 & 3.35 & & & & & \\
\hline ES_Promedio & 0.00 & 6.45 & 5.97 & 6.86 & 4.00 & 3.10 & & & & & \\
\hline
\end{tabular}

Los resultados comparativos con otros modelos de geoide indican que los valores mínimos corresponden a los modelos GRACE2014 y DEC2007. Este análisis marca una separación entre los modelos y confirma que estos son los que tienen la mayor precisión y resolución entre los comparados. Este análisis validaría nuestra hipótesis de que el modelo GeoiDEC14 es el más preciso obtenida hasta la fecha en el área de la isla Decepción. La razón de la existencia de una diferencia más pequeña respecto al modelo experimental DEC2007 y al modelo GRACE2014, podemos decir que se deben a que el primero fue obtenido con datos experimentales in situ, mientras que el modelo GRACE2014 es el modelo global más preciso obtenido hasta la fecha, resolución grado 200, que se traduce en una mayor precisión y por lo tanto sus datos se acercan más a modelos obtenidos con datos reales tomados in situ. Más detalles sobre esta comparativa de presentan en Jigena et al. (2016).

El estudio de la distribución espacial de los valores de N en GeoiDEC14, muestra que algunas de sus anomalías coinciden con los puntos calientes de la isla, i.e., los valores mínimos se ubican en bahía Fumarolas y bahía Balleneros, que son zonas con presencia de fumarolas y puntos calientes. 
Por otro lado, los valores máximos los podemos encontrar en zonas donde existen restos de flujo de lavas, i.e., Colatinas, Glaciar Negro o Punta Murature. Estas anomalías pueden deberse a las propiedades de la corteza o a la densidad del terreno en la zona (Crescentini y Amoruso, 2007), que se pueden apreciar en la geología de la isla.

\section{Conclusiones}

La Antártica es una región donde los datos de mareas son escasos, por este motivo las series temporales con más de un año de observación son muy valiosas para estudios de geofísica, oceanografia que se requieren en la zona. La importancia de este trabajo radica en que es un trabajo pionero en varios aspectos, entre ellos:

a. Es el primer estudio con registros de mareas en Decepción y Livingston con más de dos años de observación continua (798 días), corresponden a datos tomados en varias campañas antárticas en las campañas antárticas 2010-11, 2011-12, 2012-13), que ha permitido obtener 70 constituyentes de marea de corto periodo (diurno y semidiurno) y de largo período (quincenal, mensual, semi-anual y anual).

b. También es el primer trabajo donde se determina con mucha precisión el nivel medio del mar (NMM), que es el plano de referencia vertical fundamental, que es de obligada utilización en aplicaciones geodésicas, geofísicas y oceanográficas tanto técnicas como científicas, donde se requiera una referencia altimétrica precisa. Además, con este trabajo se ha iniciado la vinculación a la referencia altimétrica de la redes geodésicas de la Antártica como la RGAE, REGID y RENID, y que continuará en el futuro.

c. Es el primer modelo de un geoide local de precisión obtenido en una zona volcánica activa, como es el volcán Decepción, y en esta zona de la Antártida y que será de mucha aplicación tanto en proyectos de investigación como en actividades técnicas y de ingeniería.

Con este trabajo se subsanan las carencias de registros de marea contínuos y de largo período y que además que se encuentren vinculados a sistemas de referencia oficiales (e.g. ITRF, 2000) y referenciados altimétricamente a puntos de referencia vertical permanentes, que sean utilizados a partir de la fecha como benchmarks de referencia de mareógrafos (TGBM).

Otro resultado importante de este trabajo há sido la obtención de una altura ortométrica precisa en los vértices geodésicos de referencia (BEJC, COLA y LNO0) y la monumentación física de las marcas de referencia altimétrica como los (TGBM, Tide Gauge Benchmark) y las marcas auxiliares de referencia de nivel (TGAR, Tide Gauge Auxiliary Reference), que quedaran como datums fundamentales para referencia vertical y horizontal de las estaciones 
mareográficas de LIVMAR y DECMAR, y que utilizaremos posteriormente para la determinación del nivel medio del mar en la zona.

El modelo de geoide GEODEC14, ha sido obtenido mediante el método directa GPS-Nivelación-Gravedad, combinando datos de GPS, de nivelación y gravimetría para determinar la ondulación del geoide con la mayor precisión posible y que ha hecho posible contar con un modelo de geoide cuya precisión es superior a la de cualquier modelo global existente a la fecha e inclusive a modelos experimentales obtenidos anteriormente, como es el modelo experimental local DEC2007.

El nuevo modelo local GeoiDEC14, al tener una mayor precisión, combinado con el GPS, permite determinar las alturas ortométricas directamente y con la precisión suficiente para ser utilizado en aplicaciones de geofísica, topográfica y oceanográficas en toda el área de estudio, principalmente en la zona interior de la isla Decepción, alrededor de Puerto Foster.

El nivel medio del mar (NMM) es el plano de referencia vertical fundamental, de obligada utilización en aplicaciones geodésicas, geofísicas y oceanográficas tanto técnicas como científicas. Este trabajo es uno de los primeros de una serie de estudios de marea utilizando series de datos a largo período y que en el futuro podrá ser vinculado para la referenciación altimétrica de la Red Geodésica de Isla Decepción (REGID) y la Red Geodésica de la Antártida Española (RGAE).

Para el presente trabajo, se estudió también la posibilidad de aplicar otras metodologías para la determinación del geoide, entre ellas el método RemoveRestore, sin embargo los resultados obtenidos no fueron muy satisfactorios (Berrocoso et al., 2008). Además, este método es difícil aplicación en la zona debido a la falta de datos, i.e., Modelo Digital del Terreno (DTM), Modelo Digital de Elevación (DEM); que sería fundamental para obtener con esta metodología un modelo de geoide preciso.

Es importante señalar que este geoide experimental, GeoiDEC14, puede mejorarse en el futuro, mediante la adición de datos de la zona exterior de la isla, ya que la altura ondulación del geoide en la parte externa de la isla se obtuvo por extrapolación, tomando los valores de los puntos ubicados en la bahía interior, lo que implica una mayor error en la parte exterior de la isla. También sería de mucha ayuda añadir medidas gravimétricas marinas tanto en el interior de Puerto Foster como en la zona del estrecho de Bransfield entre las islas Livingston y Decepción. Es aconsejable que en trabajos futuros se realice un nuevo levantamiento altimétrico, con la finalidad de corregir los errores de marco de referencia altimétrico definido en 2003 y redefinir un nuevo marco de referencia con las nuevas precisiones alcanzadas.

Considerando, que hemos obtenido nuevos valores altimétricos en los puntos de referencia de la redes REGID y RENID que han mejorado el plano de referencia vertical (NMM), por lo que actualmente disponemos de un marco de referencia actualizado, es conveniente completar este estudio con datos satélite de radar altímetro de radar de las medidas instantáneas del nivel del mar. 
Por último, en isla Livingston, proporcionamos una nueva y más precisa altura ortométrica en las estaciones geodésicas BEJC y BEJ1 y en la estación de control mareógrafo TOJO. En estos puntos posteriormente también se obtuvo la ondulación del geoide (N). Los valores de $\mathrm{N}$ obtenidos en Livingston son similares al valor medio obtenido en la isla Decepción, y dado que la isla Livingston no tiene actividad volcánica y los valores de $\mathrm{N}$ son muy similares, por lo tanto no se detecta ninguna anomalía en la zona. Aunque estos tres valores obtenidos en Livingston no permiten determinar un geoide en esta pequeña zona; sin embargo, nos sirven para comparar los valores obtenidos en isla Decepción, que al ser similares nos permiten inferir que en ambas islas la estructura de corteza y la distribución de masas no varían significativamente.

\section{Agradecimientos}

La realización de esta tesis doctoral y posterior publicación de este artículo ha sido posible gracias al apoyo y la financiación de las siguientes instituciones: Universidad de Cádiz (UCA), Agencia Española de Cooperación Internacional para el Desarrollo (AECID), al Laboratorio de Astronomía, Geodesia y Cartografía de la UCA y al Grupo de Investigación RNM-314. A las tripulaciones de las Bases Antárticas Españolas "Gabriel de Castilla", isla Decepción y "Juan Carlos I", isla Livingston, y de los buques de Investigación Científica españoles "Las Palmas" y “Hespérides, por el apoyo a los trabajos de campo donde el autor participó como investigador antártico durante las Campañas Antárticas 2008-2009 y 2009-2010. A los directores de la tesis, los doctores Manuel Berrocoso Domínguez y Juan Vidal Pérez.

\section{Bibliografía}

Altamimi, Z., Sillard P. \& Boucher C. (2002). ITRF2000: A new release of the International Terrestrial Reference Frame for earth science applications. J. Geophys. Res., 107(B10), 2214. https://doi.org/10.1029/2001JB000561

Berrocoso M., Gárate J., Martín-Dávila J., Fernández-Ros A., Moreu G. \& Jigena B. (1996). Improving the local geoid with GPS. Reports of the Finnish Geodetic Institute, 96(2), 91-96, Masala. Recuperado de

https://rodin.uca.es/bitstream/handle/10498/20949/1996_2_FGI_local\%20 geoid_articulo\%2BIndice.pdf?sequence=1\&isAllowed=y

Berrocoso M., Fernández-Ros A., Torrecillas C., Enriquez-Salamanca J. M., Ramírez M. E., Pérez-Peña A., González-Fuentes M. J., Páez R., Jiménez-Teja Y., García-García A., Tárraga M. \& García-García F. (2006a). Geodetic Research on Deception Island, Antarctica. En Fütterer D. K., Damaske D., Kleinschmidt G., Miller H. \& Tessensohn F. (Eds.) Antarctica: Contributions to Global Earth Sciences, 391-396. Springer, Berlin, Heidelber https://doi.org/10.1007/3-540-32934-X_49

Berrocoso M., Jiménez Teja Y., Páez R. (2006b). Determination of a physical reference frame for Deception Island. Geophysical Research Abstracts. 
Berrocoso M., Ramírez, M. E., Fernández-Ros, A., Torrecillas, C., Enríquez-Salamanca, J. M., Pérez-Peña, A., Páez, R., Jiménez-Teja, Y., González-Fuentes, M.J., SánchezAlzola, A., García-García, A., Tárraga, M., \& García-García, F. (2006c). Diseño, desarrollo, objetivos y estado actual de las redes geodésicas establecidas en la Antártida durante las campañas antárticas españolas. VII Simposio de Estudios Polares (pp. 48-50). Granada, España.

Berrocoso, M., Salamanca J. M., Ramírez, M. E., Fernández-Ros, A. \& Jigena, B. (2007). Determination of a local geoid for Deception Island, in Antarctica: A keystone in a Changing World, Online Proceedings of the 10th ISAES X. En A. K. Cooper and C. R. Raymond (Ed.), USGS Open-File Report 2007-2007-1047, Poster Sesion 145, 141 https://doi.org/10.3133/of2007-1047

Berrocoso M., Fernández-Ros A., Ramírez M. E., Enríquez de Salamanca J. M., Torrecillas C., Pérez-Peña A., Páez R., García-García A., Jiménez-Teja Y., García-García F., Soto R., Gárate J., Martín-Dávila J., Sanchez-Alzola A., De Gil A., FernándezPrada J.A. \& Jigena B. (2008). Geodetic Research on Deception Island and its Environment (South Shetland Islands, Bransfield Sea and Antarctic Peninsula) during Spanish Antarctic Campaigns (1987-2007). En Capra A., Dietrich R. (Eds.) Geodetic and Geophysical Observations in Antarctica, 97-124. Springer, Berlin, Heidelberg. https://doi.org/10.1007/978-3-540-74882-3_6

Berrocoso M., Torrecillas C., Jigena B. \& Fernández-Ros A. (2012). Determination of geomorphological and volumetric variations in the 1970 land volcanic craters area (Deception Island, Antarctica) from 1968 using historical and current maps, remote sensing and GNSS. Antarctic Science, 24(04), 367-376. https://doi.org/10.1017/S0954102012000193

Carbó A., Muñoz-Martín A., Dávila J., Catalán M. \& García A. (2001). Análisis de nuevos datos gravimétricos marinos en el entorno de la Isla Decepción (Islas Shetland del Sur, Antártida). Revista de la Sociedad Geológica de España, 14(3-4), 189-197. Recuperado de https://sge.usal.es/archivos/REV/14(3-4)/Art03.pdf

Centro Geográfico del Ejército Español (CGE) (1992). Topographic map of Deception Island. $1: 25000$. Madrid.

Crescentini L. \& Amoruso A. (2007). Effects of crustal layering on the inversion of deformation and gravity data in volcanic areas: An application to the Campi Flegrei caldera, Italy. Geophysical Research Letters, 34, L09303,

https://doi.org/10.1029/2007gl029919

Defant A. (1961). Physical oceanography. New York: Pergamon Press.

D'onofrio E., Dragani W. C., Speroni J. O. \& Fiore M. E. (2003). Propagation and amplification of tide at the north-eastern coast of the Antarctic Peninsula. An observational study. Polar Geoscience, 16, 53-60.

Dragani W. C., Drabble M. R., D'onofrio E. E. \& Mazio C. A. (2004). Propagation and amplification of tide at Bransfield and Gerlache straits, northwestern Antarctic Peninsula. An observational study. Polar Geosciences, 17, 156-170.

Fernández-Ros A. (2006). Modelización de movimientos y deformaciones de la corteza terrestre mediante observaciones de los satélites del Sistema de Posicionamiento Global. Tesis doctoral, Departamento de Matemáticas, Facultad de Ciencias, Universidad de Cádiz. 
Fofonoff N. P. \& Millard (1983). Algorithms for computation of fundamental properties of seawater. UNESCO Technical Papers in Marine Science, 44, 53 pp.

Foreman M. G. G. (1977). Manual for tidal heights analysis and prediction. Sidney, BC: Institute of Ocean Sciences, Pacific Marine Science Report 77-10, 97 pp.

García M. A. (1994). Oceanografía dinámica de un mar Antártico: el Estrecho de Bransfield. Investigación Española en la Antártida. Seminario de la Universidad Internacional Menéndez Pelayo (pp. 193-208) Santander, 19-23 julio, 1993. Madrid: Centro de Publicaciones, Ministerio de Educación y Ciencia.

González-Ferrán, O. (1991) The Bransfield Rift and its active volcanism. En Thomson M. R. A., Crame J. A., Thomson J. W. (Eds.), Geological Evolution of Antarctica, (pp. 505-509). Cambridge University Press, Cambridge.

Gracia E., Canals M., Farranz M., Sorribas J. \& Pallas R. (1997). Central and eastern Bransfield basins (Antarctica) from high-resolution swath-bathymetry data. Antarctic Science, 9, 168-180.

Heiskanen W. A. \& Moritsz H. (1985) Physical Geodesy. Instituto Geografico Nacional. Madrid, España.

King M. A. \& Padman L. (2005). Accuracy assessment of ocean tide models around Antarctica. Geophysical Research Letters, (32), L23608 https://doi.org/10.1029/2005GL023901Ci

Jigena B., Vidal J. and Berrocoso M. (2014) Determination of the Mean Sea Level at Deception and Livingston Islands. Antarctic Science 27(01), 101-102. https://doi.org/10.1017/S0954102014000595

Jigena B., Vidal J. \& Berrocoso M. (2015). Determination of the tide constituents at Livingston and Deception Islands (South Shetland Islands, Antarctica), using annual time series. DYNA 82 (191), 209-218.

https://doi.org/10.15446/dyna.v82n191.45207

Jigena B., Berrocoso M., Torrecillas C., Vidal J., Barbero I. \& Fernandez-Ros A. (2016). Determination of an experimental geoid at Deception Island, South Shetland

Islands, Antarctica. Antarctic Science, 28(4), 277-292, https://doi.org/10.1017/S0954102015000681

Ledesma R., Macbeth G., \& Cortada N. (2008). Tamaño del efecto: revisión teórica y aplicaciones con el sistema estadístico ViSta. Revista Latinoamericana de Psicología, 40(3), 425-440. Recuperado de https://dialnet.unirioja.es/servlet/articulo?codigo=2925044

Lenn Y. D., Chereskin T. K. \& Glatts R. C. (2003). Seasonal to tidal variability in currents, stratification and acoustic backscatter in an Antarctic ecosystem at Deception Island. Deep Sea Research Part II: Topical Studies in Oceanography, 50(10-11), 1665-1683. https://doi.org/10.1016/S0967-0645(03)00085-7

López O., García M. A. \& Sánchez-Arcilla A. S. (1993). Marea y circulación en el Estrecho de Bransfield durante el verano austral 92-93. En Cacho J. \& Serrat D. (Eds.). Actas del V Simposio Español de Estudios Antárticos (pp. 389-401). Comisión Interministerial de Ciencia y Tecnología, Madrid, España.

López O., García M. A. \& Sánchez-Arcilla A. S. (1994). Tidal and residual currents in the Bransfield Strait, Antarctica. Annales Geophysicae, 12, 887-902. 
López O., García M. A., Gomis D., Rojas P., Sospedra J. \& Sánchez-Arcilla A. S. (1999). Hydrographic and hydrodynamic characteristics of the eastern basin of the Bransfield Strait (Antartica). Deep-Sea Research I, 46, 1755-1778.

Maestro A., Somoza L., Barnolas A., Rey J., Martínez-Frías J., \& López-Martínez, J. (2007). Active tectonics, fault patterns and stress field of Deception Island: A response to oblique convergence between the Pacific and Antarctic Plates. Journal of South American Earth Sciences, 23(2-3), 256-268. https://doi.org/10.1016/j.jsames.2006.09.023.

Martí J., Vila J. \& Rey J. (1996). Deception Island (Bransfield Strait, Antarctica); an example of a volcanic caldera developed by extensional tectonics. En McGuire, W. J., Jones, A. P., Neuberg, J. (Eds.). Volcano instability on the earth and other planets. Special Publications 110, 253-265. London: Geological Society, London.

Muñoz Pérez J. J., Abarca Molina J. M. (2009). Influencia del viento y de las variaciones de la presión atmosférica en el nivel del mar de marismas y estuarios. Revista de Obras Públicas, (3505), ISSN 0034-8619.

Padman L., Fricker H. A., Coleman R., Howard S. and Erofeeva L. (2002). A new tide model for the Antarctic ice shelves and seas. Annals of Glaciology, 34, 247-254. https://doi.org/10.3189/172756402781817752

Schöne T., Pohl M., Zakrajsek A.F.and Schenke H. W. (1998). Tide gauge measurements, a contribution for the long-term monitoring of the sea level. En Wiencke, Ferreyra, Arntz, Rinaldi (Eds.) The Potter Cove coastal ecosystem, Antarctica, Berichte zur Polarforschung, 299, 12-14.

SCAR (Scientific Committee for Antarctic Research) (1993). Antarctic digital database on CD-ROM. Cambridge: SCAR.

Seeber G. (2003). Satellite Geodesy, 2nd completely revised and extended edition. Walter de Gruyter GmbH \& Co. KG.

Servicio de Hidrografía Naval de la República Argentina (SHN) (1970). Informe Campaña Oceanográfica Isla Decepción 1970. Departamento de Oceanografía, Servicio de Hidrografía Naval, 70 pp. Buenos Aires, Argentina.

Smellie J. L., Liesa M., MuñozJ. A., Sabàt F., Pallàs R., \& Willam R. C. R. (1995). Lithostratigraphy of volcanic and sedimentary sequences in central Livingston Island, South Shetland Islands. Antarctic Science, 7(1), 99-113.

Smellie J. (2001). Lithostratigraphy and volcanic evolution of Deception Island, South Shetland Islands. Antarctic Science, 13(2), 188-209.

https://doi.org/10.1017/S0954102001000281

Smellie, J. L. (2002). The 1969 subglacial eruption on Deception Island (Antarctica): events and processes during an eruption beneath a thin glacier and implications for volcanic hazards. En Smellie J. L. \& Chapman M. G. (Eds.) Volcano-ice interaction on Earth and Mars. Geological Society of London, Special Publication, (202), 59-79. https://doi.org/10.1144/GSL.SP.2002.202.01.04

Smellie, J. L.; López-Martínez, J.; Headland, R. K.; Hernández-Cifuentes, F.; Maestro, A.; Millar, I. L.; Rey, J.; Serrano, E.; Somoza, L., \& Thomson, J. W. (2002) Geology and geo-morphology of Deception Island. Cambridge, British Antarctic Survey. (BAS Geomap Se-ries, Sheets 6A and 6B).

Smith Jr. K. L., Baldwin R. J., Glatts R. C., Chereskin T. K., Ruhl H., \& Lagun V. (2003). Weather, ice, and snow conditions at Deception Island, Antarctica: long time- 
series photographic monitoring. Deep Sea Research Part II: Topical Studies in Oceanography, 5(10-11), 1649-1664. https://doi.org/10.1016/S0967-0645(03)00084-5

Smithson, M. J. (1992). Pelagic tidal constants, IAPSO Publication Scientifique, No. 35. Birkenhead: IAPSO, IUGG.

Solari, M., Hervé, F., Martinod, J., Le Roux, J., Ramírez, L. \& Palacios, C. (2008). Geotectonic evolution of the Bransfield Basin, Antarctic Peninsula: Insights from analogue models. Antarctic Science, 20(2), 185-196. https://doi.org/10.1017/S095410200800093X

Speroni, J. O., Dragani, W. C., D’Onofrio, E. E., Drabble, M. R. \& Mazio, C. A. (2000). Estudio de la marea en el borde de la barrera Larsen, Mar de Weddell noroccidental. Geoacta, 25, 111.

Taylor F. W., Bevis M. G., Dalziel I. W. D., Smalley Jr. R. Frohlich C., Kendrick E., Foster J., Phillips D. \& Gudipati K. (2008). Kinematics and segmentation of the South Shetland Islands-Bransfield basin system, northern Antarctic Peninsula. Geochemistry Geophysics Geosystems, 9(4), Q04035, 10.1029/2007GC001873

Taylor, F. W., M. G. Bevis, I. W. D. Dalziel, R. Smalley Jr., C. Frohlich, E. Kendrick, J. Foster, D. Phillips, \& K. Gudipati(2008) Kinematics and segmentation of the South Shetland Islands-Bransfield basin system, northern Antarctic Peninsula, Geochem. Geophys. Geosyst, 9 (4), Q04035. https://doi.org/10.1029/2007GC001873

Torge W. (2001). Geodesy. Third Edition. Gruyter Eds. Berlin, NewYork.

Torrecillas C., Berrocoso M., García-García A. (2006). The Multidisciplinary Scientific Information Support System (SIMAC) for Deception Island. En Fütterer D.K., Damaske D., Kleinschmidt G., Miller H., Tessensohn F. (Eds.) Antarctica. 397-402. Springer, Berlin, Heidelberg. https://doi.org/10.1007/3-540-32934-X_50

UNESCO (1981). Tenth report of the joint panel on oceanographic tables and standards. Tech-nical Papers in Marine Science, 36, 25 pp.

UNESCO (1994). Intergovernmental Oceanographic Commission manual on sea level measurement and interpretation. Vol. II. Emerging technologies. UNESCO Manuals and Guides, 14, 77 pp.

Valencio D. A., Mendía J. E. \& Vilas J. F. (1979) Paleomagnetism and K-Ar age of Mesozoic and Cenozoic igneous rocks from Antarctica. Earth and Planetary Science Letters, 45(1), 61-68. https://doi.org/10.1016/0012-821X(79)90107-9

Vidal, J., Berrocoso, M., \& Fernández-Ros, A. (2012). Study of tides and sea levels at Deception and Livingston islands, Antarctica. Antarctic Science, 24(2), 193-201. https://doi.org/10.1017/S095410201100068X

Willmott V., Domack E., Padman L. \& Canals M. (2007). Glaciomarine sediment drifts from Gerlache Strait, Antarctic Peninsula. In: Hambry M., Christoffersen P., Glasser N. F., Hubbard B. (Eds.). Glacial sedimentary processes and products. IAS Special Publication. New York: Blackwells, 67-84. https://doi.org/10.1002/9781444304435.ch6 